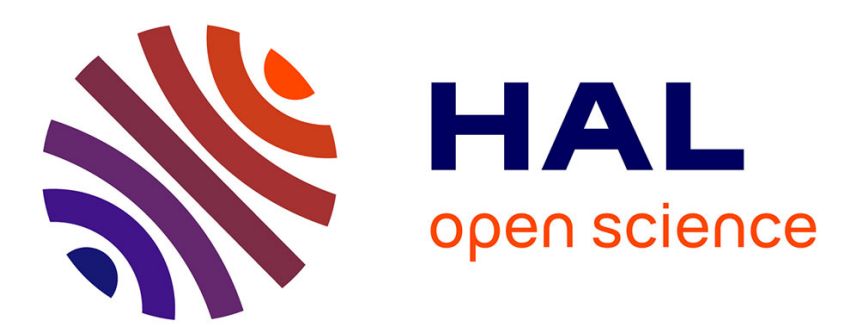

\title{
Axisymmetric contact problem for a biphasic cartilage layer with allowance for tangential displacements on the contact surface
}

I.I. Argatov, G.S. Mishuris

\section{- To cite this version:}

I.I. Argatov, G.S. Mishuris. Axisymmetric contact problem for a biphasic cartilage layer with allowance for tangential displacements on the contact surface. European Journal of Mechanics - A/Solids, 2010, 29 (6), pp.1051. 10.1016/j.euromechsol.2010.07.003 . hal-00687354

\section{HAL Id: hal-00687354 \\ https://hal.science/hal-00687354}

Submitted on 13 Apr 2012

HAL is a multi-disciplinary open access archive for the deposit and dissemination of scientific research documents, whether they are published or not. The documents may come from teaching and research institutions in France or abroad, or from public or private research centers.
L'archive ouverte pluridisciplinaire $\mathbf{H A L}$, est destinée au dépôt et à la diffusion de documents scientifiques de niveau recherche, publiés ou non, émanant des établissements d'enseignement et de recherche français ou étrangers, des laboratoires publics ou privés. 


\section{Accepted Manuscript}

Title: Axisymmetric contact problem for a biphasic cartilage layer with allowance for tangential displacements on the contact surface

Authors: I.I. Argatov, G.S. Mishuris

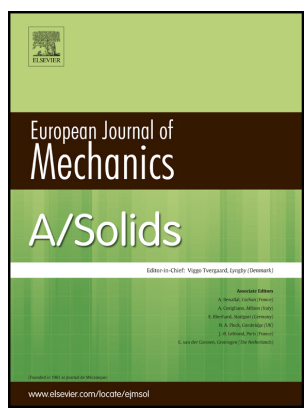

PII: $\quad$ S0997-7538(10)00094-X

DOI: $\quad$ 10.1016/j.euromechsol.2010.07.003

Reference: EJMSOL 2629

To appear in: European Journal of Mechanics / A Solids

Received Date: 6 October 2009

Revised Date: 12 July 2010

Accepted Date: 12 July 2010

Please cite this article as: Argatov, I.I., Mishuris, G.S. Axisymmetric contact problem for a biphasic cartilage layer with allowance for tangential displacements on the contact surface, European Journal of Mechanics / A Solids (2010), doi: 10.1016/j.euromechsol.2010.07.003

This is a PDF file of an unedited manuscript that has been accepted for publication. As a service to our customers we are providing this early version of the manuscript. The manuscript will undergo copyediting, typesetting, and review of the resulting proof before it is published in its final form. Please note that during the production process errors may be discovered which could affect the content, and all legal disclaimers that apply to the journal pertain. 


\title{
Axisymmetric contact problem for a biphasic cartilage layer with allowance for tangential displacements on the contact surface
}

\author{
I.I. Argatov ${ }^{\mathrm{a}}$, G.S. Mishuris ${ }^{\mathrm{b}, *}$ \\ ${ }^{a}$ Laboratory of Friction and Wear, Research Institute of Mechanical Engineering \\ Problems, V.O., Bolshoy pr., 61, 199178 St. Petersburg, Russia \\ ${ }^{\mathrm{b}}$ Institute of Mathematics and Physics, Aberystwyth University, Ceredigion SY23 \\ 3BZ, Wales, UK
}

\begin{abstract}
A unilateral axisymmetric contact problem for a biphasic cartilage layer indented by a rigid punch is considered. The refined linearized kinetic relationship which takes into account the both radial and tangential displacements of the boundary points of the biphasis cartilage layer is imposed. The obtained analytical solution is valid over long time periods and can be used for increasing loading conditions. It can be used as it is and as a benchmark for verification of FEM model accuracy.
\end{abstract}

Key words: Contact problem, cartilage layer, tangential displacements, analytical solution

\section{Introduction}

Biomechanical contact problems involving transmission of forces across biological joints are of considerable practical interest. Many solutions to the problem of contact interaction of articular cartilage surfaces in joints are available. Ateshian et al. (1994) obtained an asymptotic solution for the contact problem of two identical biphasic cartilage layers attached to two rigid impermeable spherical bones of equal radii. Wu et al. (1996) extended this solution to a more general model by combining the assumption of the kinetic relationship from classical contact mechanics (Johnson, 1985) with the joint contact model for the contact of two biphasic cartilage (Ateshian et al., 1994). An improved solution for the contact of two biphasic cartilage layers which can be used for dynamic loading was obtained by Wu et al. (1997a).

\footnotetext{
* Corresponding author.

Email addresses: argatov@home.ru (I.I. Argatov), ggm@aber.ac.uk (G.S. Mishuris).
} 
It is known (Wu et al., 2000) that in real joints, the structural and biomechanical properties of the cartilage may change dramatically in the pathologic stage. Moreover, the geometrical complexity of the problem cannot give a chance to solve it analytically in the general setting and the numerical approach (mostly with FEM) moves to the frontier of the research and becomes recently the main tool for dealing with wider spectrum of medical application (Wilson et al., 2005). It is a common knowledge, however, that the numerical simulations should be carefully verified. As in any practical problem, experimental results could be the most efficient verification. Unfortunately, in most types of biomechanical contact problems, where experiments cannot be easily performed in vivo, other methods to verify FEM models become increasingly important. And if comparison of the numerical results obtained with different FEM packages seems to be the way to do so, the most efficient way still remains a comparison with the exact analytical results. In their article, Wu et al. (1997b) compared the solutions obtained using ABAQUS with other finite element models and with those obtained using analytical solutions.

Moreover, in the case of a thin contrast interface (with properties essentially different from those in the adherents) the FEM code often fails, that is its reliable application requires so a fine mesh inside the interface and in its neighborhood (see, for example, (Mishuris and Öchsner, 2007)) that it turns out to be unrealistic to obtain any credible results especially if time dependent problems are considered. For this reason, asymptotic methods recently come back to the game, and many researchers considered various revised problems which previously have been tackled in the most simple formulations (Benveniste and Baum, 2007; Benveniste, 2009).

Relatively recently, the tangential displacements effect was found to be quite important in the nanoindentation technique for determining the elastic properties. Observing that the so-called Bulychev-Alekhin-Shorshorov relation (Bulychev et al., 1976) for analyzing nanoindentation load-displacement data does not take into account the radial displacements of material under the indenter, Hay et al. (1999) introduced the corresponding correction factor, which turned out to be Poisson's ratio dependent. For spherical indenters, Hay and Wolff (2001) have shown that the additive correction is proportional to the ratio $a / R$, where $a$ is the contact radius and $R$ the indenter radius. The refined nonlinear boundary conditions for the actual contact were discussed by Fu and Fischer-Cripps (2005). Therefore, the refined kinetic relationship which takes into account the radial tangential displacements of the boundary points of a biphasic cartilage layer should be imposed instead of the classical kinetic relationship for correct predictions of biomechanical parameters in the contact region of the articular cartilage.

The purpose of this study is to revisit the existing analytical solutions for the axisymmetric analytical contact problem of a biphasic cartilage layer with a rigid punch and to construct a new more accurate solution of the model contact problem which is also capable to represent the limit situation when the biomechanical properties of the contacting cartilage layers differ drastically. The obtained solution is valid over long time periods and under increasing loading conditions. It can be used as it is and as a benchmark for verification of FEM model accuracy.

The analytical approach proposed here is directly based on the methods previously developed by Ateshian et al. (1994), Wu et al. (1997a), and Argatov (2004). Note that analytical approaches to various contact problems related to biomechanics were developed previously by Eberhardt at al. (1991) and Haider and Holmes (1995). Recent contributions relevant to the problem under consideration approach are those of Pavliotis and Holmes (2002) and Batra and Jiang (2008). However, to the best of the authors' knowledge, this is the first time that the biomechanical 
contact problem has been solved in the refined formulation with the tangential displacements taken into account.

In solving contact problems for a punch indenting an elastic body which are formulated in the frame of the linear theory of elasticity, it is a standard assumption that the tangential displacements of the boundary points of the elastic body that come in contact with the punch are negligible (see, e.g., Gladwell (1980); Johnson (1985)). This simplified assumption is employed to uncouple the normal and tangential displacements along the contact interface, thus considerably simplifying the resulting system of integral equations which has to be solved. The contact problems with allowance for the tangential displacements at the contact zone were considered by Brock (1979), Georgiadis (1998), Argatov (2004). It was shown that the refined contact problem formulation implies new qualitative features in singular contact problems with geometrical discontinuities of the indenter (Brock, 1979; Georgiadis, 1998), whereas the tangential-displacement effects in the contact problem for two elastic bodies are proportional to the relative difference between the elastic moduli of the contacting bodies (Argatov, 2004). To the best of the authors' knowledge, this paper presents the first investigation of the tangential-displacement effects in the contact problem for a biphasic cartilage layer. The obtained results were partially presented at the 1st International Conference on Mathematical and Computational Biomedical Engineering ${ }^{1}$.

Note that, as a rule, simplifying hypotheses should allow to obtain results at least qualitatively comparable with experimental results. The simplified shape of the punch and the mechanical properties of the cartilage are justified by the aim of the study. In fact, in contrast to the simplifying geometry we introduce the refined contact condition. The numerous simplifying hypotheses which appear throughout the derivation of the approximate analytical solution are verified by the numerical calculations on the basis of the exact solution.

\section{Refined formulation of the contact problem}

We consider a thin linear biphasic cartilage layer indented by a punch shaped like a body of revolution. Introducing the cylindrical coordinate system $(r, \varphi, z)$, we write the equation of the punch surface (before loading) in the form $z=h+\Phi(r)$, where $h$ is the thickness of cartilage layer. For simplicity, we assume that the punch occupies a convex domain $z \geq h+\Phi(r)$ whereas it is in contact with the plane $z=h$ at a single point chosen as the coordinate origin. In the particular case of a punch shaped like a paraboloid of revolution, we have

$$
\Phi(r)=\frac{r^{2}}{2 R_{0}},
$$

where $R_{0}$ is the curvature radius of the punch surface at its apex.

We denote the vertical displacement of the punch by $-\delta_{0}(t)$. The unilateral contact condition that the boundary points of the cartilage layer do not penetrate into the punch can be written

1 Mishuris, G. and Argatov, I. Exact solution to a refined contact problem for biphasic cartilage layers, Proc. 1st International Conference on Mathematical and Computational Biomedical Engineering - CMBE2009 June 29 - July 1, 2009, Swansea, UK. P. Nithiarasu and R. Löhner (Eds.). P. 1-4 
as follows (Kravchuk, 1977):

$$
w(r, t)+\delta_{0}(t)-\Phi(r+u(r, t)) \leq 0 .
$$

According to Ateshian et al. (1994), the horizontal and vertical displacements of the boundary points of the tissue, $u(r, t)$ and $w(r, t)$, are expressed in the following dimensionless form:

$$
\begin{aligned}
& u\left(r^{\prime}, t^{\prime}\right)=-h \frac{\varepsilon}{2 \mu_{s}} \frac{\partial p\left(r^{\prime}, t^{\prime}\right)}{\partial r^{\prime}} \\
& w\left(r^{\prime}, t^{\prime}\right)=h \frac{\varepsilon^{2}}{\mu_{s}}\left\{\frac{1}{3} \frac{1}{r^{\prime}} \frac{\partial}{\partial r^{\prime}}\left(r^{\prime} \frac{\partial p\left(r^{\prime}, t^{\prime}\right)}{\partial r^{\prime}}\right)+\frac{1}{H} \int_{0}^{t^{\prime}} \frac{1}{r^{\prime}} \frac{\partial}{\partial r^{\prime}}\left(r^{\prime} \frac{\partial p\left(r^{\prime}, \tau\right)}{\partial r^{\prime}}\right) d \tau\right\}
\end{aligned}
$$

Here, $\varepsilon=h / \bar{a}_{0}$ is a dimensionless small parameter with $\bar{a}_{0}$ denoting a characteristic measure of the contact radius, $H=\left(\lambda_{s}+2 \mu_{s}\right) / \mu_{s}$ is a material parameter of the cartilage, where $\lambda_{s}$ and $\mu_{s}$ represent the first Lamé coefficient and the shear modulus of the solid phase of the cartilage tissue, $p\left(r^{\prime}, t^{\prime}\right)$ is the contact pressure. The dimensionless radial coordinate and time, $r^{\prime}$ and $t^{\prime}$, are defined by the formulas

$$
r^{\prime}=\frac{r}{\bar{a}_{0}}, \quad t^{\prime}=\frac{t}{\tau^{\prime}}, \quad \tau^{\prime}=\frac{h^{2}}{\left(\lambda_{s}+2 \mu_{s}\right) k},
$$

where $k$ denotes the cartilage permeability.

The equality in relation (2) determines the contact radius $a(t)$. In other words, the following equation holds within the contact area: $w(r, t)+\delta_{0}(t)-\Phi(r+u(r, t))=0$ for $r \leq a(t)$. We assume that the displacement $u(r, t)$ is small compared to the contact radius $a(t)$. In this case, the written-out nonlinear contact condition can be replaced by the following linearized kinetic relationship:

$$
w(r, t)+\delta_{0}(t)-\Phi(r)-\Phi^{\prime}(r) u(r, t)=0, \quad r \leq a(t) .
$$

Here the prime denotes differentiation.

In view of formula (1), Eq. (6) takes the form

$$
-w(r, t)+\frac{r}{R_{0}} u(r, t)=\delta_{0}(t)-\frac{r^{2}}{2 R_{0}}, \quad r \leq a(t) .
$$

Expressing the displacements $w(r, t)$ and $u(r, t)$ in the dimensional form by means of formulas $(3)-(5)$, we write the displacement compatibility condition (7) in the following form (we assume that $r \leq a(t))$ :

$$
\frac{1}{r} \frac{\partial}{\partial r}\left(r \frac{\partial p(r, t)}{\partial r}\right)+\chi \int_{0}^{t} \frac{1}{r} \frac{\partial}{\partial r}\left(r \frac{\partial p(r, \tau)}{\partial r}\right) d \tau+\varkappa r \frac{\partial p(r, t)}{\partial r}=m\left(C r^{2}-\delta_{0}(t)\right)
$$

Here we introduced the notation

$$
\chi=\frac{3 \mu_{s} k}{h^{2}}, \quad \varkappa=\frac{3}{2 h R_{0}}, \quad m=\frac{3 \mu_{s}}{h^{3}}, \quad C=\frac{1}{2 R_{0}} .
$$


Eq. (8) is used to find the contact pressure density $p(r, t)$. The radius of the contact area $a(t)$ is determined from the condition that the contact pressure is positive and vanishes at the contour of the contact area:

$$
p(r, t) \geq 0, \quad r \leq a(t) ; \quad p(a(t), t)=0 .
$$

Moreover, the contact pressure should satisfy the boundary conditions

$$
\left.\frac{\partial p(r, t)}{\partial r}\right|_{r=0}=0,\left.\quad \frac{\partial p(r, t)}{\partial r}\right|_{r=a(t)}=0
$$

where the first one comes from the standard idea for an axisymmetric blunt punch, whereas the second one appears in the case of contact problem for a biphasis cartilage layer, where it is additionally assumed a smooth transition of the surface normal stresses from the contact region $r<a(t)$ to the outside region $r>a(t)$ (Ateshian et al., 1994). Finally, the equilibrium equation for the whole system is

$$
2 \pi \int_{0}^{a(t)} p(\rho, t) \rho d \rho=F(t)
$$

where $F(t)$ denotes the external load.

For non-decreasing loads when $d F(t) / d t \geq 0$, the contact radius increases monotonously, i.e., the following condition holds (Wu et al., 1997a): $d a(t) / d t>0$.

It is readily seen from formulas (3) and (4) that the tangential displacement $w\left(r^{\prime}, t^{\prime}\right)$ is an order of magnitude greater than the normal displacement $u\left(r^{\prime}, t^{\prime}\right)$. Thus, the formulation of the contact problem excluding the tangential displacement comes in contradiction with neglecting terms that could contribute significantly in certain configurations. This consideration implies the question whether and how the tangential displacements can influence the parameters of the contact pressure distribution. It is clear that the inclusion of the tangential displacements makes the formulation of contact problem more precise though the obtained refined problem becomes much more difficult, and requires a much more elaborate approach for analytical solution.

The aim of this study is to derive a solution for the refined contact problem for a biphasic cartilage layer in the case of increasing loading condition handling accurately both radial and tangential displacements of the cartilage boundary. The solution proposed here is directly based on the approach developed by Ateshian et al. (1994), Wu et al. (1997a), and Argatov (2004).

In the next section we formulate the most important obtained results concerning asymptotic relationships between the main biomechanical parameters. In Section 3, we derive and solve equations for the punch displacement and the radius of the contact area. In Section 4, we obtain analytical representations for the contact pressure density. In Section 5, we show that the contact problem under consideration coincides in the case $\varkappa=0$ with that studied in detail by Ateshian et al. (1994), Wu et al. (1997a). Overall, this paper addresses the question when accounting of the additional term in Eq. (8) is important for applications.

Some of the technical derivations useful for understanding and further applications are included in Appendices. 


\section{Outline of main results}

Based on the governing integral equation (8), we have established two a priori relationships between the three main parameters of contact. Namely, the following equations connect the unknown radius of the contact area $a(t)$, the unknown punch displacement $\delta_{0}(t)$ and the contact load $F(t)$ :

$$
\begin{gathered}
\delta_{0}(t)=C \frac{a^{2}(t)}{2}+\frac{2 \varkappa}{m} \frac{F(t)}{\pi a^{2}(t)}, \\
\frac{\pi m}{48} C a^{6}(t)=\mathcal{K} F(t)+\varkappa\left\{\frac{a^{2}(t)}{4} F(t)+\frac{\pi}{2} \int_{0}^{a(t)} \rho^{4} \frac{\partial p(\rho, t)}{\partial \rho} d \rho\right\} .
\end{gathered}
$$

Here we have introduced the following short hand notation for the Volterra integral operator $\mathcal{K}$ on the right-hand side of Eq. (14) and its inverse $\mathcal{K}^{-1}$ :

$$
\mathcal{K} F(t)=F(t)+\chi \int_{0}^{t} F(\tau) d \tau, \quad \mathcal{K}^{-1} f(t)=f(t)-\chi \int_{0}^{t} f(\tau) e^{-\chi(t-\tau)} d \tau
$$

In a specific case $\varkappa=0$ Eqs. (13) and (14) coincide with the results obtained by Ateshian et al. (1994); Wu et al. (1997a). We underline that these equations are exact consequences of the governing integral equation (8).

In order to solve Eq. (14) and derive an approximation for the radius of the contact area, we have invented a new asymptotic method based on the recursive relation for the integral characteristics of the contact pressure density. The following equation takes into account the first order correction to the effect of tangentional displacements on the contact radius:

$$
\frac{\pi m}{48} C\left(a^{6}(t)+\frac{\varkappa}{4} \mathcal{K}^{-1} a^{8}(t)\right)=\mathcal{K} F(t)+\frac{\varkappa a^{2}(t)}{4} F(t) .
$$

In the domain $0<r<a_{0}$, the following approximate formula for the derivative of the contact pressure distribution takes place:

$$
\begin{aligned}
\frac{\partial p^{-}}{\partial r}(r, t) & =\frac{m r}{8}\left\{2 C r^{2} e^{-\chi t}-4 \mathcal{K}^{-1} \delta_{0}(t)\right. \\
& \left.+\varkappa r^{2}\left(\frac{C}{3} r^{2} e^{-\chi t}(\chi t-1)+\mathcal{K}^{-2} \delta_{0}(t)\right)\right\}+O\left(\varkappa^{2}\right), \quad \varkappa \rightarrow 0 .
\end{aligned}
$$

In the interval $a_{0}<r<a(t)$, we have the following asymptotic representation of the derivative of the contact pressure distribution:

$$
\begin{aligned}
\frac{2 r}{m} \frac{\partial p^{+}}{\partial r}(r, t) & =\langle a(t)-r\rangle\left\{\frac{C}{2} a_{0}^{2}\left(2 r^{2}-a_{0}^{2}\right) e^{-\chi t}-r^{2} \mathcal{K}^{-1} \delta_{0}(t)\right. \\
& \left.+\frac{\varkappa}{4}\left(r^{4} \mathcal{K}^{-2} \delta_{0}(t)+\frac{1}{3} C a_{0}^{2}(\chi t-1)\left(3 r^{4}-3 r^{2} a_{0}^{2}+a_{0}^{4}\right) e^{-\chi t}\right)\right\} \\
& +R(r, t, \varkappa)+O\left(\varkappa^{2}\right), \quad \varkappa \rightarrow 0 .
\end{aligned}
$$


Here $\langle\cdot\rangle$ is the Heaviside step function defined in the standard way: $\langle x\rangle=1$ if $x \geq 0$ and $\langle x\rangle=0$ if $x<0$ and the auxiliary integral $R(r, t, \varkappa)$ is determined by formulas (61) and (62).

The main difficulty in calculating the contact pressure is the variability of the contact area that complicates the application of the Laplace transform. A sophisticated numerical algorithm have been elaborated for dealing with integral equations with variable domain.

\section{Equations for the punch displacement and the radius of the contact area}

Let us integrate Eq. (8) with respect to $r$ and transform the obtained integral using integration by parts. Since the constant of integration $D_{1}(t)$ vanishes in accordance with the regularization condition $(11)_{1}$, we get

$$
\mathcal{K} \frac{\partial p(r, t)}{\partial r}+\varkappa\left(r p(r, t)-\frac{2}{r} \int_{0}^{r} p(\rho, t) \rho d \rho\right)=m\left(C \frac{r^{3}}{4}-\delta_{0}(t) \frac{r}{2}\right),
$$

which, after substituting the value $r=a(t)$ and taking into account the boundary conditions (10), (11) as well as the monotonicity condition $d a(t) / d t>0$, transforms into

$$
-\frac{2 \varkappa}{a(t)} \int_{0}^{a(t)} p(\rho, t) \rho d \rho=m\left(C \frac{a^{3}(t)}{4}-\delta_{0}(t) \frac{a(t)}{2}\right) .
$$

Finally, in view of formula (12), Eq. (21) takes the form of Eq. (13).

We now return to Eq. (20) which after integration with respect to variable $r$ take the forms:

$$
\mathcal{K} p(r, t)+\varkappa\left\{\int_{0}^{r} p(\rho, t) \rho d \rho-2 \int_{0}^{r} p(\rho, t) \rho \ln \frac{r}{\rho} d \rho\right\}=m\left(C \frac{r^{4}}{16}-\delta_{0}(t) \frac{r^{2}}{4}\right)+D_{2}(t) .
$$

By using the boundary condition (10), the constant of integration can be obtained as a function of the contact radius $a(t)$ :

$$
D_{2}(t)=\frac{m}{4} \delta_{0}(t) a^{2}(t)-\frac{m}{16} C a^{4}(t)+\frac{\varkappa}{2 \pi} F(t)-2 \varkappa \int_{0}^{a(t)} p(\rho, t) \rho \ln \frac{a(t)}{\rho} d \rho .
$$

Multiplying both sides of Eq. (22) by $r$ and integrating over the contact radius, we obtain

$$
\begin{aligned}
\frac{1}{2 \pi} \mathcal{K} F(t) & +\varkappa\left\{\int_{0}^{a(t)} \rho \int_{0}^{\rho} p(\xi, t) \xi d \xi d \rho-2 \int_{0}^{a(t)} \rho \int_{0}^{\rho} p(\xi, t) \xi \ln \frac{\rho}{\xi} d \xi d \rho\right\} \\
& =m\left(C \frac{a^{6}(t)}{96}-\delta_{0}(t) \frac{a^{4}(t)}{16}\right)+\frac{a^{2}(t)}{2} D_{2}(t) .
\end{aligned}
$$


We change the order of integration in the second and third integrals in (24). After that, taking into account formulas (12), (23), and (13), we transform Eq. (24) into the following equation:

$$
\frac{\pi m}{48} C a^{6}(t)=\mathcal{K} F(t)+\varkappa\left\{\frac{a^{2}(t)}{4} F(t)-2 \pi \int_{0}^{a(t)} p(\rho, t) \rho^{3} d \rho\right\} .
$$

For further convenience we rewrite condition (25) in the equivalent form (14). Notice that Eq. (14) was obtained by integrating by parts in the integral with taking into account the condition $(10)_{2}$.

\section{Contact pressure}

Returning to Eq. (8), we introduce a new dependent variable $y(r, t)$ such that

$$
y(r, t)=r \frac{\partial p(r, t)}{\partial r} .
$$

Using the substitution (26), we represent Eq. (8) in the form

$$
\left.\frac{1}{r} \frac{\partial y(r, t)}{\partial r}+\chi \int_{0}^{t} \frac{1}{r} \frac{\partial y(r, \tau)}{\partial r} d \tau+\varkappa y(r, t)=m f(r, t)\right) .
$$

where

$$
f(r, t)=\langle a(t)-r\rangle\left(C r^{2}-\delta_{0}(t)\right)
$$

This allows us to rewrite the equation in the form:

$$
\frac{1}{r}\left(1+\frac{\chi}{s}\right) \frac{d \tilde{y}}{d r}+\varkappa \tilde{y}=m \tilde{f}(r, s)
$$

where the tilde denotes the time Laplace transform and $s$ is the Laplace transform variable. Note that all these functions should be analytic in the half-plane $\Re s>0$.

Taking into account the regularity condition $(11)_{1}$, which now reads $\tilde{y}(r, s)=O\left(r^{2}\right)$ as $r \rightarrow 0$, we obtain the corresponding particular solution of Eq. (29) for arbitrary $r>0$ :

$$
\tilde{y}(r, s)=\frac{m}{\varkappa} e^{-K(s) \frac{r^{2}}{2}} \int_{0}^{r} e^{K(s) \frac{\xi^{2}}{2}} \xi K(s) \tilde{f}(\xi, s) d \xi .
$$

Here we introduced the notation

$$
K(s)=\frac{\varkappa s}{s+\chi}
$$

Under the assumption utilized in this paper that the loading is monotonic (as a result, $a(t)$ is an increasing function) one can compute $\tilde{f}(r, s)$ to have: 


$$
\tilde{f}(r, s)=\left\{\begin{array}{c}
\frac{C r^{2}}{s}-\tilde{\delta}_{0}(s), \quad 0 \leq r \leq a_{0}, \quad b(r)=\left\{\begin{array}{c}
0, \quad 0 \leq r \leq a_{0}, \\
\frac{C r^{2} e^{-s b(r)}}{s}-\int_{b(r)}^{\infty} e^{-s t} \delta_{0}(t) d t, \quad r \geq a_{0} .
\end{array} \quad a_{0} \leq r .\right.
\end{array}\right.
$$

where we denote by $a^{-1}(r)$ the inverse to the function $a(t)$.

Please include Figure 1 somewhere here.

Not that the solution to Eq. (27) is different from zero only in the domain $\Omega_{-} \bigcup \Omega_{+}$drawn on Fig. 1. We shall consider this problem in the both domains separately.

\subsection{Solution of Eq. (27) in domain $\Omega_{-}$}

Let us denote by $y^{-}(r, t)$ the solution of Eq. (27) in the domain $\Omega_{-}\left(0 \leq r \leq a_{0}(0)\right)$. After some algebra, we can rewrite Eq. (30) in the interval $0 \leq r \leq a_{0}$ as follows:

$$
\frac{\varkappa}{m} \tilde{y}^{-}(r, s)=\frac{C r^{2}}{2 s}+\left(\frac{2 C}{s K(s)}+\tilde{\delta}_{0}(s)\right)\left(\exp \left(-K(s) \frac{r^{2}}{2}\right)-1\right) .
$$

Taking into account the asymptotic estimate $\tilde{y}(r, s)=O\left(r^{2}\right)$ as $r \rightarrow 0$, we obtain

$$
\frac{\varkappa}{m} \frac{\partial \tilde{p}^{-}(r, s)}{\partial r}=\frac{C r}{s}+\left\{\frac{2 C}{s K(s)}+\tilde{\delta}_{0}(s)\right\}\left\{\exp \left(-\frac{\varkappa r^{2}}{2}\right) \frac{1+\tilde{L}(s, r)}{r}-\frac{1}{r}\right\} .
$$

Here we have introduced the following notations:

$$
\tilde{L}(s, r)=\exp \{G(s, r)\}-1, \quad G(s, r)=\frac{\varkappa \chi r^{2}}{2(s+\chi)} .
$$

Then the direct inverse Laplace transform formula gives

$$
\begin{aligned}
\frac{\varkappa}{m} \frac{\partial p^{-}(r, t)}{\partial r} & =C r+\frac{\exp \left(-\frac{\varkappa r^{2}}{2}\right)-1}{r}\left[\frac{2 C}{\varkappa}(1+\chi t)+\delta_{0}(t)\right] \\
& +\frac{1}{r} \exp \left(-\frac{\varkappa r^{2}}{2}\right) \int_{0}^{t} L(t-\tau, r)\left[\frac{2 C}{\varkappa}(1+\chi \tau)+\delta_{0}(\tau)\right] d \tau
\end{aligned}
$$

The inverse to the function $\tilde{L}(s, r)$ is calculated in the following form:

$$
L(t, r)=\frac{1}{2 \pi i} \int_{-i \infty+\eta}^{i \infty+\eta}(\exp \{G(s, r)\}-1) e^{s t} d s,
$$

where $-\chi<\eta \leq 0$ is an arbitrary number. Properties of this function and discussion of the methods for its effective computation can be found in Appendix 1. Using (70), one can check that the condition (11) is valid for any solution determined by Eq. (34). 
Now, we can compute pressure distribution at any particular time $t=t_{*}$ employing formula (36). However, for large time this equation is not the best representation from the numerical point of view. In fact, as it was shown in (53), the contact area increases with time to be estimated as $a(t)=O\left(t^{1 / 6}\right)$ as $t \rightarrow \infty$. Then, from Eq. (13) we get $a(t)=O\left(t^{1 / 3}\right)$ as $t \rightarrow \infty$ for the fixed total load $F(t)=F_{0}$. Then the second and the third terms in the solution (36) tend separately to infinity as $t \rightarrow \infty$, whereas the pressure distribution, $p(r, t)$, has to decrease with time, because the total contact load $F_{0}$ is fixed. To confirm this we integrate in the integral in (36) by parts with taking into account identity (40) to obtain

$$
\begin{aligned}
\frac{\varkappa}{m} \frac{\partial p^{-}(r, t)}{\partial r} & =C r+\left[\frac{\exp \left(-\frac{\varkappa r^{2}}{2}\right)-1}{r}\right. \\
& \left.-\frac{1}{r} M(0, r) \exp \left(-\frac{\varkappa r^{2}}{2}\right)\right]\left(\frac{2 C}{\varkappa}(1+\chi t)+\delta_{0}(t)\right) \\
& +\frac{1}{r} M(t, r) \exp \left(-\frac{\varkappa r^{2}}{2}\right)\left(\frac{2 C}{\varkappa}+\delta_{0}(0)\right) \\
& +\frac{1}{r} \exp \left(-\frac{\varkappa r^{2}}{2}\right) \int_{0}^{t} M(t-\tau, r)\left(\frac{2 C \chi}{\varkappa}+\delta_{0}^{\prime}(\tau)\right) d \tau
\end{aligned}
$$

Here the function $M(t, r)$ is defined similarly to the kernel function $L(t, r)$ :

$$
M(t, r)=\frac{1}{2 \pi i} \int_{-i \infty-\eta}^{i \infty-\eta} \frac{1}{s}(\exp \{G(s, r)\}-1) e^{s t} d s .
$$

In fact, there is the close relationship between them:

$$
\frac{\partial}{\partial t} M(r, t)=L(r, t)
$$

Properties of the function $M(t, r)$ are discussed in Appendix 2. With use of (75), Eq. (38) can be simplified as follows:

$$
\begin{aligned}
\frac{\varkappa}{m} \frac{\partial p^{-}(r, t)}{\partial r} & =C r+M(t, r) \exp \left(-\frac{\varkappa r^{2}}{2}\right)\left[\frac{2 C}{\varkappa}+\delta_{0}(0)\right] \\
& +\frac{1}{r} \exp \left(-\frac{\varkappa r^{2}}{2}\right) \int_{0}^{t} M(t-\tau, r)\left[\frac{2 C \chi}{\varkappa}+\delta_{0}^{\prime}(\tau)\right] d \tau .
\end{aligned}
$$

One can see that, in contrast with the representation (36), all the terms in Eq. (41) are bounded as $t \rightarrow \infty$.

Note that Eqs. (36) and (41) have formally sense not only in the interval $0 \leq r \leq a_{0}$ but for any $r \in(0, a(t))$. However, this fact does not mean that they necessarily represent the solution of Eq. (27) for any given function $\delta_{0}(t)$ in the whole domain $0 \leq r \leq a(t)$. For this reason, we have to return back to Eq. (27) and consider it in the interval $a_{0} \leq r \leq a(t)$ independently.

\subsection{Solution of Eq. (27) in domain $\Omega_{+}$}

If $r \geq a_{0}$, Eq. (30) can be rewritten in the following form using integrating by parts: 


$$
\begin{aligned}
\frac{\varkappa}{m} \tilde{y}_{+}(r, s) & =\tilde{f}(r, s)-e^{-\varkappa \frac{r^{2}}{2}} e^{G(s, r)} \tilde{f}(0, s) \\
& -e^{-K(s) \frac{r^{2}}{2}} \int_{0}^{a_{0}} e^{K(s) \frac{\xi^{2}}{2}} \frac{\partial}{\partial \xi} \tilde{f}(\xi, s) d \xi \\
& -e^{-K(s) \frac{r^{2}}{2}} \int_{a_{0}}^{r} e^{K(s) \frac{\xi^{2}}{2}} \frac{\partial}{\partial \xi} \tilde{f}(\xi, s) d \xi
\end{aligned}
$$

Note that

$$
\frac{\partial}{\partial \xi} \tilde{f}(\xi, s)=\frac{2 C \xi}{s}, \quad 0 \leq \xi \leq a_{0},
$$

and the first integral in (42) can be computed to obtain

$$
\begin{aligned}
\frac{\varkappa}{m} \tilde{y}_{+}(r, s) & =\tilde{f}(r, s)-e^{-\varkappa \frac{r^{2}}{2}} e^{G(s, r)} \tilde{f}(0, s) \\
& -\frac{2 C}{s K(s)}\left[e^{-\varkappa \frac{r^{2}-a_{0}^{2}}{2}} e^{G\left(s, \sqrt{r^{2}-a_{0}^{2}}\right)}-e^{-\varkappa \frac{r^{2}}{2}} e^{G(s, r)}\right] \\
& -\int_{a_{0}}^{r} e^{-\varkappa \frac{r^{2}-\xi^{2}}{2}} e^{G\left(s, \sqrt{r^{2}-\xi^{2}}\right)} \frac{\partial}{\partial \xi} \tilde{f}(\xi, s) d \xi
\end{aligned}
$$

To calculate the remaining integral in $(44)$, we need first to find the derivative $\tilde{f}_{\xi}^{\prime}(\xi, s)$ in the interval $r>a_{0}$. From Eq. (32) it follows that

$$
\frac{\partial}{\partial \xi} \tilde{f}(\xi, s)=e^{-s b(\xi)}\left[\frac{2 C \xi}{s}-b^{\prime}(\xi)\left(C \xi^{2}-\delta_{0}(b(\xi))\right)\right] .
$$

The following identities can be established by direct integration

$$
\begin{aligned}
\frac{1}{2 \pi i} \int_{-i \infty+\eta}^{i \infty+\eta} e^{s t} \frac{\partial}{\partial \xi} \tilde{f}(\xi, s) d s \\
=\left\{\begin{array}{cc}
2 C \xi, & t>b(\xi) \\
0, & 0 \leq t \leq b(\xi)
\end{array}\right\}-b^{\prime}(\xi)\left(C \xi^{2}-\delta_{0}(b(\xi))\right) \delta(t-b(\xi)) \\
=\left\{\begin{array}{cc}
2 C \xi, & \xi<a(t) \\
0, & \xi \geq a(t)
\end{array}\right\}-b^{\prime}(\xi)\left(C \xi^{2}-\delta_{0}(t)\right) \delta(\xi-a(t)) .
\end{aligned}
$$

Here $\delta(x)$ is the Dirac delta function. In the domain $t>b(\xi)$, we have

$$
\begin{aligned}
\frac{1}{2 \pi i} \int_{-i \infty+\eta}^{i \infty+\eta} e^{s t}\left(e^{G\left(s, \sqrt{r^{2}-\xi^{2}}\right)}-1\right) \frac{\partial}{\partial \xi} \tilde{f}(\xi, s) d s \\
=2 C \xi\left(e^{\varkappa \frac{r^{2}-\xi^{2}}{2}}-1+M\left(t-b(\xi), \sqrt{r^{2}-\xi^{2}}\right)\right) \\
-b^{\prime}(\xi)\left(C \xi^{2}-\delta_{0}(b(\xi))\right) L\left(t-b(\xi), \sqrt{r^{2}-\xi^{2}}\right) .
\end{aligned}
$$


Notice that this function is equal to zero when $t \leq b(\xi)$ or that is equivalent when $\xi \geq a(t)$.

It remains now to invert Eq. (44) with taking into account (45) and (47). For $a_{0}<r<a(t)$, we have

$$
\begin{gathered}
\frac{\varkappa r}{m} \frac{\partial p^{+}}{\partial r}(r, t)=C r^{2}-\delta_{0}(t)+e^{-\varkappa \frac{r^{2}}{2}}\left\{\frac{2 C}{\varkappa}(1+\chi t)\right. \\
\left.+\delta_{0}(t)+\int_{0}^{t} L(t-\tau, r)\left[\frac{2 C}{\varkappa}(1+\chi \tau)+\delta_{0}(\tau)\right] d \tau\right\} \\
-\frac{2 C}{\varkappa} e^{-\varkappa \frac{r^{2}-a_{0}^{2}}{2}}\left\{(1+\chi t)+\int_{0}^{t} L\left(t-\tau, \sqrt{r^{2}-a_{0}^{2}}\right)(1+\chi \tau) d \tau\right\} \\
-2 C \int_{a_{0}}^{r} e^{-\varkappa \frac{r^{2}-\xi^{2}}{2}} \xi M\left(t-b(\xi), \sqrt{r^{2}-\xi^{2}}\right) d \xi \\
+\int_{a_{0}}^{r} e^{-\varkappa \frac{r^{2}-\xi^{2}}{2}} L\left(t-b(\xi), \sqrt{r^{2}-\xi^{2}}\right) \times \\
b^{\prime}(\xi)\left(C \xi^{2}-\delta_{0}(b(\xi))\right) d \xi-C\left(r^{2}-a_{0}^{2}\right) .
\end{gathered}
$$

Taking advantage from asymptotic estimate (70), one can check that

$$
y_{-}\left(a_{0}-, t\right)=y_{+}\left(a_{0}+, t\right), \quad \frac{\partial y_{-}}{\partial r}\left(a_{0}-, t\right)=\frac{\partial y_{+}}{\partial r}\left(a_{0}+, t\right)
$$

We also can rewrite the solution representation (48) for $r>a_{0}$ in the same way as it has been done in (41) for $0<r<a_{0}$. We have

$$
\begin{aligned}
& \frac{\varkappa}{m} \frac{\partial p^{+}}{\partial r}=\frac{C}{r} a_{0}^{2}+\frac{1}{r} e^{-\varkappa \frac{r^{2}}{2}}\left\{M(t, r)\left[\frac{2 C}{\varkappa}+\delta_{0}(0)\right]\right. \\
& \left.+\frac{1}{r} \int_{0}^{t} M(t-\tau, r)\left[\frac{2 C \chi}{\varkappa}+\delta_{0}^{\prime}(\tau)\right] d \tau\right\} \\
& -\frac{2 C}{\varkappa r} e^{-\varkappa \frac{r^{2}-a_{0}^{2}}{2}}\left\{M\left(t, \sqrt{r^{2}-a_{0}^{2}}\right)+\chi \int_{0}^{t} M\left(t-\tau, \sqrt{r^{2}-a_{0}^{2}}\right) d \tau\right\} \\
& \quad-\frac{2 C}{r} \int_{a_{0}}^{r} e^{-\varkappa \frac{r^{2}-\xi^{2}}{2}} \xi M\left(t-b(\xi), \sqrt{r^{2}-\xi^{2}}\right) d \xi \\
& +\frac{1}{r} \int_{a_{0}}^{r} e^{-\varkappa \frac{r^{2}-\xi^{2}}{2}} L\left(t-b(\xi), \sqrt{r^{2}-\xi^{2}}\right) b^{\prime}(\xi)\left(C \xi^{2}-\delta_{0}(b(\xi))\right) d \xi
\end{aligned}
$$




\subsection{Asymptotics of the initial contact problem and some qualitative conclusions}

Expanding the expression for the initial contact pressure $p(r, 0) \equiv p^{-}(r, 0)$, where $p^{-}(r, 0)$ is given by formula (87), into the power series of the parameter $\varkappa$, we obtain

$$
\begin{aligned}
\frac{1}{m} p(r, 0) & =\frac{\delta_{0}(0)}{4}\left(a_{0}^{2}-r^{2}\right)-\frac{C}{16}\left(a_{0}^{4}-r^{4}\right) \\
& -\varkappa\left\{\frac{\delta_{0}(0)}{32}\left(a_{0}^{4}-r^{4}\right)-\frac{C}{144}\left(a_{0}^{6}-r^{6}\right)\right\}+O\left(\varkappa^{2}\right) .
\end{aligned}
$$

In view of formula (90), we can rewrite the obtained asymptotics as follows:

$$
\frac{1}{m} p(r, 0)=\frac{C}{16}\left(a_{0}^{2}-r^{2}\right)^{2}\left\{1+\frac{\varkappa a_{0}^{2}}{36}\left(1-4 \frac{r^{2}}{a_{0}^{2}}\right)\right\}+O\left(\varkappa^{2}\right) .
$$

It is easy to show that under the non-burdensome condition $\varkappa a_{0}^{2} \leq 12$ the initial contact pressure given by formula (51) is positive. Observe that the contact pressure $p(r, 0)$ in the central region tends to increase with the parameter $\varkappa$, whereas the accounting for the tangential displacements leads to a decrease in the contact pressure in the boundary region of contact.

Further, expanding the right-hand side of Eq. (93) into the power series of the parameter $\varkappa$, we find

$$
\frac{2 \pi}{m} \int_{0}^{a_{0}} p(\rho, 0) \rho^{3} d \rho=\frac{\pi C a_{0}^{8}}{192}\left(1-\frac{7 \varkappa a_{0}^{2}}{20}\right)+\frac{\varkappa F_{0} a_{0}^{4}}{12 m}+O\left(\varkappa^{2}\right) .
$$

Substituting this asymptotic expansion into Eq. (92), after some algebra, we obtain

$$
\frac{\pi m}{48} C a_{0}^{6}=F_{0}+\varkappa^{2} F_{0} \frac{a_{0}^{4}}{240}+O\left(\varkappa^{3}\right)
$$

We stress that the first-order term is absent in Eq. (52). This explains the paradoxical fact that formula (52) predicts a small increase in the initial radius of contact while at the same time the contact pressure at the central region of contact also increases with the increase in the parameter $\varkappa$.

Since we consider a monotonic loading (the function $F(t)$ does not decrease with time), $a(t)$ will increases up to infinity what proves by contradiction. Assuming reasonable growth of the function $F(t)$ that is $F(t) \rightarrow F_{\infty}$ as $t \rightarrow \infty$, the following estimate holds true:

$$
a(t) \sim\left(\frac{48 \chi F_{\infty} t}{\pi m C}\right)^{1 / 6}, \quad t \rightarrow \infty
$$

As a result, one can write asymptotic expansion for the other sought-for function $\delta_{0}(t)$ :

$$
\delta_{0}(t) \sim\left(\frac{48 \chi F_{\infty} t}{\pi m C}\right)^{1 / 3}, \quad t \rightarrow \infty,
$$

Finally, from formula (90) it is easy to see that the accounting for the tangential displacements results in an increase in the punch displacement $\delta_{0}(0)$. 


\section{Approximate solution to the problem for arbitrary time}

In the previous three sections we have found basic equations solving the problem under consideration. They respectively are: (13), (25), (41), (50). Unfortunately, they require substantial computational efforts to reconstruct the exact numerical solution. However, in a particular case when the parameter $\varkappa$ in its dimensionless form is reasonably small, an approximate solution to the problem will provide acceptable answer. Notice that equation (13) defining the punch indentation $\delta_{0}(t)$ does not need any simplification.

\subsection{Approximate equation for the radius of the contact area}

In order to obtain an approximation for the radius of the contact area, we have derived the recursive relation for the integral characteristics of the contact pressure density (see Appendix 3). In particular, for $n=1$, Eq. (84) takes the form

$$
\mathcal{K} F_{1}(t)=\frac{\pi m C a^{8}(t)}{192}+\varkappa\left\{-\frac{a^{4}(t)}{24} F_{0}(t)+\frac{3}{8} F_{2}(t)\right\}
$$

where $F_{n}(t), n=0,1,2$, are integral characteristics of the contact pressure density determined by formula (78).

Neglecting the last term in the right-hand side of Eq. (55), we derive

$$
F_{1}(t)=\frac{\pi m C}{192} \mathcal{K}^{-1} a^{8}(t)
$$

Now, substituting the expression (56) into the right-hand side of Eq. (79), we arrive at the approximate equation for the radius of the contact area (16).

In principle, the resolving equation for $a(t)$ can be made more accurate by employing the interactive process based upon formula (84).

For example, using formulas (55) and (84), $n=2$, we have

$$
F_{1}(t)=\frac{\pi m C}{192} \mathcal{K}^{-1} a^{8}(t)+\frac{\varkappa}{24} \mathcal{K}^{-1}\left\{-a^{4}(t) F(t)+\frac{3 \pi m C}{160} \mathcal{K}^{-1} a^{10}(t)\right\} .
$$

It is readily seen that formula (57) refines formula (56) by means of the second order terms and leads to

$$
\begin{aligned}
& \frac{\pi m}{48} C\left(a^{6}(t)+\frac{\varkappa}{4} \mathcal{K}^{-1} a^{8}(t)+\frac{3 \varkappa^{2}}{80} \mathcal{K}^{-2} a^{10}(t)\right) \\
& =\mathcal{K} F(t)+\frac{\varkappa}{4} a^{2}(t) F(t)+\frac{\varkappa^{2}}{24} \mathcal{K}^{-1}\left(a^{4}(t) F(t)\right) .
\end{aligned}
$$

Note that for $t=0$ the condition (16) transforms with taking into account evident identities $\mathcal{K} f(0)=f(0)$ and $\mathcal{K}^{-1} f(0)=f(0)$ into the equation

$$
\frac{\pi m C}{48 F(0)} a_{0}^{6}=1
$$


which is consistent with Eq. (25).

\subsection{Approximate formula for the contact pressure}

Let us consider the limit value (as $\varkappa \rightarrow 0$ ) of Eq. (36) in the domain $0<r<a_{0}$. We can show that

$$
\begin{aligned}
& \frac{\varkappa}{m} \frac{\partial p^{-}(r, 0)}{\partial r}=C r-\left(\frac{\varkappa r}{2}-\frac{\varkappa^{2} r^{3}}{8}\right)\left[\frac{2 C}{\varkappa}(1+\chi t)+\delta_{0}(t)\right] \\
& +\frac{1}{r}\left(1-\frac{\varkappa r^{2}}{2}\right) \int_{0}^{t} L(t-\tau, r)\left[\frac{2 C}{\varkappa}(1+\chi \tau)+\delta_{0}(\tau)\right] d \tau+O\left(\varkappa^{2}\right), \quad \varkappa \rightarrow 0 .
\end{aligned}
$$

After some algebra with use of (70), we obtain

$$
\begin{aligned}
& \frac{2}{m r} \frac{\partial p^{-}(r, t)}{\partial r}=-\delta_{0}(t)+\chi \int_{0}^{t} e^{(t-\tau) \chi} \delta_{0}(\tau) d \tau \\
& \quad+\frac{1}{2} C r^{2} e^{-\chi t}+\frac{\varkappa r^{2}}{4}\left(\frac{C}{3} r^{2} e^{-\chi t}(\chi t-1)+\delta_{0}(t)\right. \\
& \left.\quad-\chi \int_{0}^{t} e^{(t-\tau) \chi}(2-\chi(t-\tau)) \delta_{0}(\tau) d \tau\right)+O\left(\varkappa^{2}\right) .
\end{aligned}
$$

In the interval $a_{0}<r<a(t)$, the asymptotic representation of the pressure should be found from Eq. (50). After some algebra, we arrive at formula (18) with $R(r, t, \varkappa)=R_{1}(r, t, \varkappa)+R_{2}(r, t, \varkappa)$ and

$$
\begin{aligned}
R_{1}(r, t, \varkappa)= & C \int_{a_{0}}^{r}\langle a(t)-\xi\rangle\left(r^{2}-\xi^{2}\right) e^{-\chi(t-b(\xi))} \times \\
& \left(2 \xi+\frac{1}{2} \varkappa(\chi(t-b(\xi))-1) \xi\left(r^{2}-\xi^{2}\right)\right) d \xi \\
R_{2}(r, t, \varkappa)= & \chi \int_{0}^{b(r)}\langle a(t)-a(\tau)\rangle\left(r^{2}-a^{2}(\tau)\right) e^{-\chi(t-\tau)}\left(C a^{2}(\tau)-\delta_{0}(\tau)\right) \times \\
& \left(1-\frac{\varkappa}{4}\left(r^{2}-a^{2}(\tau)\right)(2-\chi(t-\tau))\right) d \tau .
\end{aligned}
$$

It is evident that $R\left(a_{0}, t, \varkappa\right)=0$ and $R_{r}\left(a_{0}, t, \varkappa\right)=0$. Thus, the matching conditions (49) are valid in the main terms for the asymptotic expansion. It is also important to note that substituting (17), (18) into (14) and collecting the leading order terms, after some algebra, one 
can recover Eq. (100). The following two identities are of great help:

$$
\begin{aligned}
& \chi \int_{a_{0}}^{a(t)} \xi^{n} e^{-\chi(t-b(\xi))} b^{\prime}(\xi) d \xi=a^{n}(t)-\mathcal{K}^{-1} a^{n}(t) \\
& n \int_{a_{0}}^{a(t)} \xi^{n-1} e^{-\chi(t-b(\xi))} d \xi=\mathcal{K}^{-1} a^{n}(t)-a_{0}^{n} e^{-\chi t}
\end{aligned}
$$

\section{$6 \quad$ Numerical results and discussions}

\subsection{General remarks.}

In order to illustrate the constructed solution of the refined biomechanical contact problem and compare it with that from (Wu et al., 1997a), we will adopt for the computations the same material properties and geometrical parameters of a typical human cartilage reported in (Ateshian et al., 1994; Wu et al., 1997a). Namely we assume that $H_{A}=0.5 \mathrm{MPa}, \mu_{s}=0.25 \mathrm{MPa}$, $\nu=0.0, H=H_{A} / \mu_{s}=2(1-\nu) /(1-2 \nu)=2.0, k=2 \cdot 10^{-3} \mathrm{~mm}^{4} \mathrm{~N}^{-1} \mathrm{~s}^{-1}, h=1 \mathrm{~mm}$, $R_{0}=400 \mathrm{~mm}$. In the sequel we also consider some variation of this set to analyze the sensitivity of the solution to the variation of material characteristics. In all the numerical tests, a constant contact load $F(t)=F_{0}=100 \mathrm{~N}$ is applied.

Before to present the numerical results themselves, we would like to shortly discuss the computational models adapted here. First, for the reason of comparison, we compute (a) the classic solution reported in (Ateshian et al., 1994; Wu et al., 1997a) represented here by substituting $\varkappa=0$ in Eqs. (13), (25) (to compute the radius of the contact zone, $a(t)$, and the punch indentation, $\left.\delta_{0}(t)\right)$, alongside with Eqs. (17) and (18) and the boundary condition (10) to reconstruct the pressure distribution, $p(r, t)$. Note here that the last formulae were presented in (Wu et al., 1997a) in an implicit form.

Then we compute the complete numerical solution in general case $(\varkappa>0)$ according to formulas (13), (14), (41), and (50) together with the boundary condition (10). We utilize here an iterative procedure. All the values of interest: the radius of the contact zone, $a(t)$, the punch indentation, $\delta_{0}(t)$, and the contact pressure, $p(r, t)$; are computed then in the same common scheme. The procedure appears to be highly unstable and sensitive to variations of the parameters under consideration. We explain this phenomena later. Moreover, since all the parameters in question should be computed at each iteration step, the procedure large amount of memory in comparison with other computational procedures.

Finally, the solution we employ here is the approximation of the previous one with an accuracy of $O\left(\varkappa^{2}\right)$, and the solution that is constructed by means of the exact formula (13) and the approximate ones (16), (17) and (18) together with the boundary condition (10). This solution has all advantages of the classic solution described above (Wu et al., 1997a) and simultaneously involves non zero values of the parameter $\varkappa$, or, in other words, accounts for the influence of the tangential displacements in the model. 
Two crucial questions should be answered now. First, whether one should take into account the tangential displacements in the cartilage layer model. And, second, if the answer on the first question is positive, whether one can use for this reason the approximate model instead of the exact one.

To answer the first question, we observe that the small parameter $\varepsilon$ enters the right-hand sides of formulas (3) and (4) as $\varepsilon$ and $\varepsilon^{2}$, respectively. Thus, the radial tangential displacement becomes more asymptotically important with respect to the vertical normal displacement as $\varepsilon \rightarrow 0$. However, real biomechanical contact problems should be investigated for finite values of their parameters.

Now, let us rewrite the governing integral equation (8) in the dimensionless form. Denoting a characteristic measure of the contact radius by $\bar{a}_{0}$ and employing formulas (5), we obtain

$$
\frac{1}{r^{\prime}} \frac{\partial}{\partial r^{\prime}}\left(r^{\prime} \frac{\partial p^{\prime}\left(r^{\prime}, t^{\prime}\right)}{\partial r^{\prime}}\right)+\frac{1}{H} \int_{0}^{t^{\prime}} \frac{1}{r^{\prime}} \frac{\partial}{\partial r^{\prime}}\left(r^{\prime} \frac{\partial p^{\prime}\left(r^{\prime}, \tau\right)}{\partial r^{\prime}}\right) d \tau+\epsilon r^{\prime} \frac{\partial p^{\prime}\left(r^{\prime}, t^{\prime}\right)}{\partial r^{\prime}}=C \bar{a}_{0} r^{\prime 2}-\delta_{0}^{\prime}\left(t^{\prime}\right) .
$$

We introduced the following standard normalised notation (Ateshian et al., 1994; Wu et al., 1997a)

$$
p^{\prime}\left(r^{\prime}, t^{\prime}\right)=\frac{1}{m \bar{a}_{0}^{3}} p(r, t), \quad \delta_{0}^{\prime}\left(t^{\prime}\right)=\frac{1}{\bar{a}_{0}} \delta_{0}(t), \quad \epsilon=\bar{a}_{0}^{2} \varkappa=\frac{3 \bar{a}_{0}^{2}}{2 h R_{0}} .
$$

Here we have taken into account the second formula (9). Finally, taking into account the notation $\varepsilon=h / \bar{a}_{0}$, we obtain

$$
\epsilon=\frac{3}{2 \varepsilon} \frac{\bar{a}_{0}}{R_{0}}
$$

Thus, formula (67) shows that the new dimensionless parameter $\epsilon$ turns out to be inversely proportional to the parameter $\varepsilon$. At the same time, the value of $\epsilon$ is proportional to the ratio $\bar{a}_{0} / R_{0}$, which is usually small in real biomechanical contacts. In particular, in the considered example, one can put $\bar{a}_{0}=10 \mathrm{~mm}$ (Ateshian et al., 1994; Wu et al., 1997a), obtaining $\varepsilon=0.1$ and $\epsilon=0.375$, what cannot be considered as negligible value. Moreover, the ratio $\bar{a}_{0} / R_{0}$ can range in sufficiently wide limits in the experiments on evaluation of material properties by means of indentation. On the other hand, the fact that $\epsilon=0.375$ does not necessary mean that this should influence greatly all the parameters of the biomechanical contact under consideration.

It is clear that if $\epsilon$ tends to zero, the obtained exact solution (as well as the approximate one) approaches the solution given by Ateshian et al. (1994), Wu et al. (1997). It is interesting to note that we cannot calculate exactly the value of $\epsilon$ unless we solve the refined contact problem, since this parameter depends on the characteristic measure of the contact radius, $\bar{a}_{0}$, which is not given before hand. However, one can estimate the value of the parameter $\epsilon$ using the solution of the original contact problem. Using formulas (10), (27), and (110), we obtain for the case of the external load under consideration the following relation:

$$
\frac{3 a^{2}(t)}{2 h R_{0}} \simeq 3.25\left(\frac{F_{0}}{\mu_{s} R_{0}^{2}}\right)^{1 / 3}\left(1+\frac{3 \mu_{s} k}{h^{2}} t\right)^{1 / 3}
$$

For the material properties and geometrical parameters of a typical human cartilage reported in (Ateshian et al., 1994; Wu et al., 1997), the second factor on the right-hand side of formula (68) 
is less than 1.1 at the moment of time $t=200 \mathrm{~s}$. From formula (68) it is obvious what physical circumstances correspond to diminishing the influence of the tangential displacements effect.

Note also that comparing (65) with (8) one can rewrite all the results obtained above in the dimensionless form that is more convenient for numerical computations.

\subsection{Numerical comparison with the classic solution.}

Let us first compute and compare the initial contact zone $a_{0}=a(0)$ using the aforementioned models. This gives us

$$
\begin{gathered}
a_{0}^{(c l)} \simeq 10.8481 \mathrm{~mm}, \quad a_{0}^{(e x)} \simeq 10.8494 \mathrm{~mm}, \\
a_{0}^{(a p 1)} \simeq 10.8481 \mathrm{~mm}, \quad a_{0}^{(a p 2)} \simeq 10.8496 \mathrm{~mm} .
\end{gathered}
$$

Here the first two values were obtained from the first two aforementioned models, while the next two correspond to the same approximate model where $a_{0}^{(a p 1)}$ is the consequence of Eq. (16) which is an approximation of the exact solution with accuracy of $O\left(\epsilon^{2}\right)$ (in the normilised form), whereas $a_{0}^{(a p 2)}$ was computed from Eq. (25) (or equivalently (79)) together with (57) that gives the next order of approximation $O\left(\epsilon^{3}\right)$ as has been mentioned in Section 5.1.

From the first glance these computations may look surprising and need some explanations. First, according to the equation (59) we should expect that the value $a_{0}^{(a p 1)}$ computed from the first order approximate equation (16) gives the same result as that in (Wu et al. (1997a)). This means that, apart of the fact that for arbitrary time the solution differs only on term $O(\epsilon)$, at the initial moment the estimate becomes $O\left(\epsilon^{2}\right)$ instead. This, in fact, has been accurately proven in (52). However, for the value $\epsilon=0.375$ even this argument could not completely explain the extremely small difference between the obtained numerical results. And only if one looks at all equations related to the asymptotic solution with respect to the parameter $\varkappa$ (see, for example, (51) - (52), (17), and (18)), it becomes clear that the value $\varkappa a_{0}^{2} / 4=\epsilon / 4$ appears there instead of $\epsilon$ in the governing equation (65).

Other useful observation concerns the contact zone $a^{(c l)}(t)$ computed according to the classic solution (Ateshian et al., 1994; Wu et al., 1997a), which always gives the lower estimate for the accurate solution $a^{(e x)}(t)$ while that computed according to the second order approximation, $a^{(a p 2)}(t)$, always gives the upper estimate. Moreover, the difference between the latter solution and the exact one is negligible at least within reasonable range of parameters appearing in biomechanical contact problem. For this reason we have used this specific approximate model $\left(a^{(a p 2)}(t) \cong a^{(e x)}(t)\right)$ for further computations. Let us remind that the exact solution requires disproportional numerical efforts. Possible errors caused by such replacement are of the order $O\left(\epsilon^{2}\right)$ according to our estimate or even less as our computations indicated.

In Figs 2-5, we present a comparison of the results obtained for the radius of the contact zone, $a(t)$, punch indentation, $\delta_{0}(t)$, contact pressure, $p(r, t)$, and its derivative with respect to the radial coordinate $r$, for three stages of deformation corresponding to the moments $t=0,100,200$ [s]. Computations have been performed for different sets of material and geometrical parameters reported earlier (see Table 1). All curves computed according to the classic model are presented by solid lines whereas the corrected results are given by dashed lines. 
Please include Figure 2 somewhere here.

It is clear from the results presented in Fig. 2 that the difference between the obtained solution to the refined problem, which takes into account the radial tangential displacements, and the classic one (Ateshian et al., 1994; Wu et al., 1997a) increases with time. However, this difference is very small. For example, for the radius of the contact zone, $a(t)$, the discrepancy between the solutions leads to errors of $0.014 \%$ for $t=0 \mathrm{~s}$ and $0.074 \%$ for $t=200 \mathrm{~s}$. However, accounting for the radial tangential displacements leads to the higher impact upon the values of the two most important for application parameters: the punch indentation, $\delta_{0}(t)$, and the contact pressure, $p(r, t)$.

Please include Figure 3 somewhere here.

In fact, the punch indentation, $\delta_{0}(t)$, differs for these two models under investigation at the beginning and the end of the indentation process like $3.57 \%$ and $3.13 \%$, respectively. Note here that we depicted on the figures the doubled punch indentation, $2 \delta_{0}(t)$, for reason of the direct comparison with results reported in (Wu et al., 1997a). As concerns the pressure, the difference takes its maximum value at the central point $r=0$. For the set of the parameters under consideration we computed the maximum difference of contact pressures as $0.85 \%$ and $3.57 \%$ for the moments $t=0 \mathrm{~s}$ and $t=200 \mathrm{~s}$, respectively. As it has been mentioned above, the refined contact model always yields higher contact pressure maximum.

Please include Figure 4 somewhere here.

Summarizing, for the set of parameters discussed above, the difference between the classic solution and the refined one is not essential, but for the punch indentation and the contact pressure should be important in some cases though. These cases may include inverse problems of determining biomechanical and the geometrical parameters of the cartilage layer from experiments.

This situation may change even drastically for other set of biomechanical parameters as they essentially vary with progress of osteoarthritis (Wu et al., 2000). To illustrate this circumstance, we choose two other sets of parameters corresponding to a "diseased" cartilage. The second set differs from that discussed above only by one parameter - the cartilage permeability, $k$. Namely, following Wu et al. (2000), we adopt now the bigger value $k^{\prime}=4 k$. The corresponding numerical results are presented in Fig. 3. All other notation used remains the same.

As in the previous case, the computed results for the radius of the contact zone, $a(t)$, are close enough with the difference estimated as $0.014 \%$ for $t=0 \mathrm{~s}$ and $0.595 \%$ for $t=200 \mathrm{~s}$. Although at the end of the indentation process the difference even for this parameter becomes pronounced.

Please include Figure 5 somewhere here. 
The punch indentation deviates from the classic solution with practically the same accuracy $3.57 \%$ and $3.24 \%$ at the beginning and the end of the computations. Finally, the contact pressure, $p(0, t)$, differs rather essential at the end of the indentation process. The respective values are $0.82 \%$ and $4.73 \%$.

Then we consider the third set of biomechanical and geometrical parameters from the table 1 . Now three parameters changed their values (Wu et al., 2000): the thickness of the cartilage layer $h^{\prime}=2 h$, the permeability $k^{\prime}=2 k$ and the shear modulus $\mu_{s}^{\prime}=0.5 \mu_{s}$, while all other parameters are the same as in (Ateshian et al., 1994; Wu et al., 1997a).

The corresponding results presented in Fig. 4 are in the same convention as above. In this particular case, the trend is different. Indeed, in contrast to the previous two cases, the difference between the radii of the contact zone stays practically constant with time (varies from $0.0215 \%$ to $0.0213 \%$ ).

The punch indentation, $\delta_{0}(t)$, exhibits similar tendency slightly changing with time while the difference between the classic solution and that obtained in the frame of the refined model slowly decreases (from $4.465 \%$ to $4.266 \%$ ). But the most interesting conclusion concerns the contact pressure, $p(r, t)$. Namely the difference between the models stays practically without changing $(0.954 \%$ for $t=0 \mathrm{~s}$ and $0.962 \%$ for $t=200 \mathrm{~s})$. Moreover, contrary to the previous cases, the variation of the pressure itself remains practically the same during the indentation process, whatever the model is used.

Finally, we consider one set of biomechanical and geometrical parameters which is also slightly different with respect to the first one. Namely, now the thickness of the cartilage layer assumed to be smaller, $h^{\prime}=h / 2$, while all other parameters have the same values as in the case of healthy cartilage layer from (Ateshian et al., 1994; Wu et al., 1997a).

Corresponding results presented in Fig. 5. The computed results for the radius of the contact zone, $a(t)$, show the maximal error at level $0.6 \%$ for $t=200 \mathrm{~s}$ that is similar to the second set of parameters discussed above. The same conclusion is valid for the contact pressure with the discrepancy of $5 \%$. However, in this case, the difference between the values of the indentation parameter, $2 \delta_{0}(t)$, computed according to the classical and refined asymptotic models is much higher. Namely, it now equal to $9 \%$. The last example shows that it is impossible to estimate a priori the maximum difference as we have discussed above from the analytical considerations. On the other hand, we would like to underline this again that the exact solution for the refined contact problem lies somewhere between those two computed by the asymptotical models. In other words, the difference between the solutions gives the aforementioned estimate.

\section{Main conclusions}

The present study results in the analytical solution to the refined unilateral axisymmetric contact problem for a biphasic cartilage layer. It has been shown that the approximate solution (represented by formulas (13), (58), (17), and (18)) is of the same complexity as the classic one constructed by Ateshian et al. (1994); Wu et al. (1997a). Moreover, the asymptotical models not only allow to avoid computations based on the accurate exact result complicated though, but 
also to determine an accuracy of the approximate computations.

The results of numerical simulations have been summarized in Table 1.

Table 1

Maximum difference between the two models on the time interval $t \in[0,200 \mathrm{~s}]$

\begin{tabular}{cccccc}
\hline$\mu_{s}$ & $k$ & $h$ & Error in & Error in & Error in \\
$\mathrm{MPa}$ & $\mathrm{mm}^{4} \mathrm{~N}^{-1} \mathrm{~s}^{-1}$ & $\mathrm{~mm}$ & $a(t)$ & $p(r, t)$ & $\delta_{0}(t)$ \\
\hline 0.25 & $2 \cdot 10^{-3}$ & 1 & $0.07 \% \%$ & $3.57 \%$ & $3.57 \%$ \\
0.25 & $8 \cdot 10^{-3}$ & 1 & $0.59 \%$ & $4.73 \%$ & $3.57 \%$ \\
0.125 & $4 \cdot 10^{-3}$ & 2 & $0.02 \%$ & $0.96 \%$ & $4.46 \%$ \\
0.25 & $2 \cdot 10^{-3}$ & 0.5 & $0.6 \%$ & $5 \%$ & $9 \%$ \\
\hline
\end{tabular}

The refined solution always leads to some increase in the radius of the contact zone, the punch indentation as well as in the contact pressure at the center of the contact zone. This, in turns, according to the constructed solution, means that the gradient of the contact pressure is also higher than that in the classic model. Utilization of this refined solution in analysis of contact problems for a biphasic cartilage layer is desirable if one investigates different stages of osteoarthritis when biomechanical and geometrical parameters vary rather significantly. However, it may be even more important to take account of the refined contact problem formulation in solving an inverse problem of determining biomechanical and geometrical parameters of cartilage layers from experiments.

It should be noticed that the limitations of the refined formulation of contact problem to biphasic isotropic materials, axisymmetric configurations, and monotonic loadings is something that can be dealt with using numerical models. The developed approach can be generalized for nonaxisymmetric configurations as well as for non-monotonic loadings. However, the introduction of a new material model for articular cartilage (see the rather extensive review by Wilson et al. (2005)) essentially changes the governing integral equation, and to solve it analytically is a real challenge.

Finally, we stress that that from the geometrical point of view, the formulation of the contact problem is too simplified. However, the simplified geometry does not impose an obstacle for studying the effect of tangential displacements. On the other hand, to estimate this effect by means of FEM simulations is more difficult since we do not know a priori the governing nondimensional parameter controlling this effect. The employed simplifying hypotheses allow us to obtain relatively simple analytical results that can be used for estimating the effect of tangential displacements in solving the contact problems with geometries closer to the reality by means of a finite element method. 


\section{Acknowledgment}

The authors would like to express their gratitude to the Referees for their helpful comments and discussions.

\section{References}

Ateshian, G. A., Lai, W.M., Zhu, W.B. \& Mow, V.C., 1994. An asymptotic solution for the contact of two biphasic cartilage layers. Journal Biomechanics 27, 1347-1360.

Wu, J.Z., Herzog, W. \& Ronsky, J., 1996. Modeling axi-symmetrical joint contact with biphasic cartilage layers - An asymptotic solution. Journal Biomechanics 29, 1263-1281.

Johnson, K. L., 1985. Contact Mechanics. Cambridge, UK, Cambridge University Press.

Wu, J. Z., Herzog, W. \& Epstein, M., 1997. An improved solution for the contact of two biphasic cartilage layers. Journal Biomechanics 30, 371-375.

Wu, J.Z., Herzog, W. \& Epstein, M., 2000. Joint contact mechanics in the early stages of osteoarthitis. Medical Engineering and Physics 22, 1-12.

Wilson, W., van Donkelaar, C. C., van Rietberger, R. \& Huiskes, R., 2005. The role of computational models in the search for the mechanical behaviour and damage mechanisms of articular cartilage. Medical Engineering and Physics 27, 810-826.

Wu, J.Z., Herzog, W. \& Epstein, M., 1997. Evaluation of the finite element software ABAQUS for biomechanical modelling of biphasic tissues. Journal of Biomechanics 31, 165-169.

Mishuris, G., Öchsner, A., 2007. 2D modelling of a thin elasto-plastic interphase between two different materials: Plane strain case. Composite Structures 80, 361-372.

Benveniste, Y., Baum, G., 2007. An interface model of a graded three-dimensional anisotropic curved interphase, Proc. Roy. Soc. Lond. A, 463, 419-434.

Benveniste, Y., 2009. An interface model for a three-dimensional curved thin piezoelectric interphase between two piezoelectric media. Mathematics and Mechanics of Solids, 14, 102-122.

Bulychev, S.I., Alekhin, V.P., Shorshorov, M.Kh. \& Ternovskii, A.P., 1976. Mechanical properties of materials studied from kineticdiagrams of load versus depth of impression during microimpression. Strength Mater. 8, 1084-1089.

Hay, J.C., Bolshakov, A. \& Pharr, G.M., 1999. Critical examination of the fundamental relations used in the analysis of nano-indentation data. J. Mater. Res. 14, 2296-2305.

Hay, J.L., Wolff, P.J., 2001. Small correction required when applying the Hertzian contact model to instrumented indentation data. J. Mater. Res. 16, 1280-1286.

Fu, G., Fischer-Cripps, A.C., 2005. On Sneddon's boundary conditions used in the analysis of nanoindentation data. J. Mater. Sci. 40, 1789-1791.

Argatov, I. I., 2004. Approximate solution of an axisymmetric contact problem with allowance for tangential displacements on the contact surface. Journal of Applied Mechanics and Technical Physics 45, 118-123.

Eberhardt, A. W., Lewis, J.L., Keer, L. M., 1991. Normal contact of elastic spheres with two elastic layers as a model of joint articulation. J. Biomech. Eng. 113, 410-417.

Haider, M. A., Holmes, M. H., 1995. Indentation of a thin compressible elastic layer: Approximate analytic and numerical solutions for rigid flat indenters. Journal of the Mechanics and Physics of Solids 43, 1199-1219.

Pavliotis, G.A., Holmes, M.H., 2002. A perturbation-based numerical method for solving a 
three-dimensional axisymmetric indentation problem. Journal of Engineering Mathematics $43,1-17$.

Batra, R.C., Jiang, W., 2008. Analytical solution of the contact problem of a rigid indenter and an anisotropic linear elastic layer. International Journal of Solids and Structures 45, 5814-5830.

Gladwell, G. M.L., 1980. Contact Problems in the Classical Theory of Elasticity. Sijthoff and Noordhoff, Alphen aan den Rijn.

Brock, L. M., 1979. Frictionless indentation by a rigid wedge: The effect of tangential displacements in the contact zone. International Journal of Engineering Science 17, 365-372.

Georgiadis, L. M., 1998. Tangential-displacement effects in the wedge indentation of an elastic half-space - an integral-equation approach. Computational Mechanics, 21, 347-352.

Argatov, I. I., 2004. Axisymmetric Hertz problem with tangential displacements on the contact surface. Doklady Physics, 49, 222-225.

Kravchuk, A.S., 1977. On the Hertz problem for linearly and nonlinearly elastic bodies of finite dimensions. Journal of Applied Mathematics and Mechanic 41, 320-328.

\section{Appendix}

\section{A1. Kernel function $L(t, r)$. Evaluation and properties}

At first glance the function $L(t, r)$ can be easily computed from (37) as the integrand tends to zero at infinity under properly chosen value of the parameter $\eta$. Moreover, it has only one singular point $s=-\chi$ in the entire complex domain. However, this point is not a pole but an essential singularity, and an attempt to compute the corresponding residuum leads to an infinite series that is not the most convenient way for computations.

On the other hand, the integral (37) can be easily computed numerically (if $\eta=0$ is chosen, it gives, in fact, the standard Fourier transform). To improve the quality of the computation one can rewrite the formula in the equivalent form

$$
\begin{aligned}
L(t, r)= & \frac{1}{2 \pi i} \int_{-i \infty}^{i \infty}\left(e^{G(s, r)}-1-G(s, r)-\frac{1}{2} G^{2}(s, r)\right) e^{s t} d s \\
& +\frac{1}{8} \varkappa \chi r^{2}\left(4+\varkappa \chi r^{2} t\right) e^{-\chi t} .
\end{aligned}
$$

Moreover, instead of the simple choice $\eta=0$ in (37) and (69), it is always better to take in computations a negative value of the parameter $\eta$. For example, we take $\eta=-\chi / 2$.

For some limiting values of the problem parameters we can work out the asymptotics of the kernel $L(t, r)$. Thus, using the reasoning like in (69) we can derive the asymptotic expansion as $r \rightarrow 0$ for any fixed value $t$ separated from zero (i. e., $t \geq t_{0}>0$ ):

$$
L(t, r)=\frac{1}{2} r^{2} \varkappa \chi\left(1+\frac{r^{2} \varkappa \chi t}{4}+\frac{\left(r^{2} \varkappa \chi t\right)^{2}}{48}\right) e^{-\chi t}+O\left(\varkappa^{4} r^{7}\right) .
$$


Note that this asymptotics is uniformly valid for any positive $t$.

To estimate behaviour of the kernel $L(t, r)$ near the point $t=0$, we rewrite Eq. (37) in a slightly different form than Eq. (69):

$$
\begin{aligned}
L(t, r) & =\frac{1}{2 \pi i} \int_{-i \infty}^{i \infty}\left(e^{G(s, r)}-1-G(s, r)-\frac{1}{2} G^{2}(s, r)\right)\left(e^{s t}-1\right) d s \\
& +\frac{1}{8} \varkappa \chi r^{2}\left(4+\varkappa \chi r^{2} t\right) e^{-\chi t} \frac{1}{2 \pi i} \int_{-i \infty}^{i \infty}\left(e^{G(s, r)}-1-G(s, r)-\frac{1}{2} G^{2}(s, r)\right) d s .
\end{aligned}
$$

As it follows from the residuum theorem, the last integral is identically equal to zero, while the first integral gives the worst estimated term

$$
L(t, r)=\frac{1}{2} \varkappa \chi r^{2}+O(t), \quad t \rightarrow 0
$$

which is consistent with $(70)$.

Another possibility to effectively compute the kernel function $L(t, r)$ is to deform the integration line in (37) into a unit circle with a center located at the singular point. This gives:

$$
L(t, r)=\frac{1}{2 \pi} e^{-\chi t} \int_{-\pi}^{\pi}\left(e^{\frac{\varkappa \chi r^{2}}{2} e^{-i \phi}}-1\right) e^{e^{i \phi} t+i \phi} d \phi
$$

\section{A2. Kernel function $M(t, r)$. Evaluation and properties}

Apart of the fact that there is the simple relationship (40) between the kernel functions $L(t, r)$ and $M(t, r)$ the last one should be computed from (39) or with the help of following equivalent formula:

$$
\begin{aligned}
M(t, r) & =\frac{1}{2 \pi i} \int_{-i \infty-\eta}^{i \infty-\eta}\left(e^{G(s, r)}-1-G(s, r)-\frac{1}{2} G^{2}(s, r)\right) e^{s t} \frac{d s}{s} \\
& -\frac{1}{8} \varkappa r^{2}\left(4+\varkappa r^{2}(1+\chi t)\right) e^{-\chi t} .
\end{aligned}
$$

Here, $0<\eta<\chi$. As $\varkappa \rightarrow 0$, the latter formula immediately gives the following asymptotic expansion:

$$
M(t, r)=-\frac{\varkappa r^{2}}{2} e^{-\chi t}\left(1+\varkappa \frac{r^{2}}{4}(1+\chi t)\right)+O\left(\varkappa^{3} r^{6}\right) .
$$

The asymptotic formula (74) is valid for any time separated from zero (i. e., $0<t_{0} \leq t$ ). Moreover, this asymptotics holds true also when $r \rightarrow 0$. To evaluate the asymptotic bahaviour of the function $M(t, r)$ as $t \rightarrow 0$, we rewrite (73) in the form

$$
\begin{aligned}
M(t, r) & =\frac{1}{2 \pi i} \int_{-i \infty-\eta}^{i \infty-\eta}\left(e^{G(s, r)}-1-G(s, r)\right)\left(e^{s t}-1\right) \frac{d s}{s} \\
& -\frac{1}{2} \varkappa r^{2} e^{-\chi t}+\frac{1}{2 \pi i} \int_{-i \infty-\eta}^{i \infty-\eta}\left(e^{G(s, r)}-1-G(s, r)\right) \frac{d s}{s},
\end{aligned}
$$


where $0<\eta<\chi$. The first integral can be easily estimated to give $O(t)$, while the second one can be evaluated in the closed form using the residuum theorem at point $s=0$ :

$$
M(t, r)=1-e^{\frac{\varkappa r^{2}}{2}}+O(t), \quad t \rightarrow 0
$$

Another representation of the kernel function $M(t, r)$ useful for practical computation is

$$
M(t, r)=\frac{e^{-\chi t}}{2 \pi} \int_{-\pi}^{\pi}\left(e^{\varkappa r^{2} e^{-i \phi}}-1\right) e^{\frac{\chi t e^{i \phi}+2 i \phi}{2}} \frac{d \phi}{e^{i \phi}-2} .
$$

Finally, the following identity for the operators involving the kernel functions $L(t, r)$ and $M(t, r)$ can be established:

$$
\begin{aligned}
& q(r, t)+\int_{0}^{t} L(t-\tau, r) q(r, \tau) d \tau=e^{-\varkappa \frac{r^{2}}{2}} q(r, t) \\
& +M(t, r) q(r, 0)+\int_{0}^{t} M(t-\tau, r) q(r, \tau) d \tau
\end{aligned}
$$

\section{A3. Recursive relations for the integral characteristics of the contact pressure density}

Let us introduce the generalized polar moments of inertia of the contact pressure by means of the formula

$$
F_{n}(t)=2 \pi \int_{0}^{a(t)} p(\rho, t) \rho^{2 n+1} d \rho
$$

Thus, Eq. (25) can be written in the form

$$
\frac{\pi m}{48} C a^{6}(t)=\mathcal{K} F_{0}(t)+\varkappa\left\{\frac{a^{2}(t)}{4} F_{0}(t)-F_{1}(t)\right\} .
$$

Now, taking into account formula (23), we represent Eq. (22) in the following form:

$$
\begin{aligned}
& \mathcal{K} p(r, t)+\varkappa\left\{-\int_{r}^{a(t)} p(\rho, t) \rho d \rho+2 \int_{0}^{a(t)} p(\rho, t) \rho \ln \frac{a(t)}{\rho} d \rho-2 \int_{0}^{r} p(\rho, t) \rho \ln \frac{r}{\rho} d \rho\right\} \\
& =\frac{m C}{16}\left(a(t)^{2}-r^{2}\right)^{2}+\left(a(t)^{2}-r^{2}\right) \frac{\varkappa F_{0}(t)}{2 \pi a^{2}(t)} .
\end{aligned}
$$

The following formulas can be verified by direct calculations using an obvious change of the integration variable:

$$
2 \pi \int_{0}^{a(t)} \rho^{2 n+1} \int_{r}^{a(t)} p(\rho, t) \rho d \rho d r=\frac{F_{n+1}(t)}{2(n+1)}
$$




$$
\begin{aligned}
& 2 \pi \int_{0}^{a(t)} r^{2 n+1} \int_{0}^{r} p(\rho, t) \rho \ln \frac{r}{\rho} d \rho d r \\
& =\frac{2 \pi a^{2 n+2}(t)}{2(n+1)} \int_{0}^{a(t)} p(\rho, t) \rho \ln \frac{a(t)}{\rho} d \rho-\frac{a^{2 n+2}(t)}{4(n+1)^{2}} F_{0}(t)+\frac{F_{n+1}(t)}{4(n+1)^{2}}, \\
& 2 \pi \int_{0}^{a(t)} r^{2 n+1} \int_{0}^{a(t)} p(\rho, t) \rho \ln \frac{a(t)}{\rho} d \rho d r=\frac{2 \pi a^{2 n+2}(t)}{2(n+1)} \int_{0}^{a(t)} p(\rho, t) \rho \ln \frac{a(t)}{\rho} d \rho .
\end{aligned}
$$

Now, multiplying the both sides of Eq. (80) by $2 \pi r^{2 n+1}$ and integrating with respect to $r$, we obtain the following equation in view of formulas (81) - (83) and the notation (15):

$$
\begin{aligned}
& F_{n}(t)=\frac{\pi m C}{8(n+1)(n+2)(n+3)} \mathcal{K}^{-1} a^{2 n+6}(t) \\
& +\varkappa \mathcal{K}^{-1}\left\{-\frac{a^{2 n+2}(t)}{2(n+1)^{2}(n+2)} F_{0}(t)+\frac{(n+2)}{2(n+1)^{2}} F_{n+1}(t)\right\} .
\end{aligned}
$$

\section{A4. Short-time asymptotics}

Now we can analyze two important limit cases. First, suppose that $t \rightarrow 0$. Then we can immediately conclude from the behaviour of the kernel of the integral operator (37) that

$$
\frac{\varkappa}{m} \frac{\partial p^{-}(r, 0)}{\partial r}=C r+\frac{\exp \left(-\frac{\varkappa r^{2}}{2}\right)-1}{r}\left[\frac{2 C}{\varkappa}+\delta_{0}(0)\right] .
$$

It is important to check whether this solution satisfy also condition (11). Substituting $r=a_{0} \equiv$ $a(0)$, we have the following identity to be valid:

$$
C a_{0}^{2}=\left(1-\exp \left(-\frac{\varkappa a_{0}^{2}}{2}\right)\right)\left[\frac{2 C}{\varkappa}+\delta_{0}(0)\right] .
$$

Next, we determine the distribution of the pressure along the contact area at $t=0$ integrating Eq. (85). Taking into account the boundary condition (10), we find

$$
\frac{\varkappa}{m} p^{-}(r, 0)=\frac{C}{2}\left(r^{2}-a_{0}^{2}\right)+\left[\frac{2 C}{\varkappa}+\delta_{0}(0)\right] g\left(r, a_{0}, \varkappa\right),
$$

where the following new notation has been introduced:

$$
g(r, b, \varkappa)=\int_{b}^{r} \frac{\exp \left(-\frac{\varkappa \xi^{2}}{2}\right)-1}{\xi} d \xi
$$


Multiplying the both parts of the latter equation by $r$ and integrating within the interval $\left(0, a_{0}\right)$, we obtain

$$
\frac{\varkappa}{\pi m} F_{0}=-\frac{C}{4} a_{0}^{4}+\left[\frac{2 C}{\varkappa}+\delta_{0}(0)\right]\left(\frac{\exp \left(-\frac{\varkappa a_{0}^{2}}{2}\right)-1}{\varkappa}+\frac{a_{0}^{2}}{2}\right) .
$$

Note that Eqs. (86) and (89) constitute a system of nonlinear equations allowing to determine two parameters $a_{0}=a(0)$ and $\delta_{0}(0)$.

Below we will show that this approach is equivalent to that discussed in Sections 2 and 3. In fact, eliminating the exponential term from Eqs. (86) and (89) we obtain

$$
\delta_{0}(0)=C \frac{a_{0}^{2}}{2}+\frac{2 \varkappa}{m} \frac{F_{0}}{\pi a_{0}^{2}} .
$$

Notice that Eq. (90) coincides with (13) for $t=0$. Substituting now this equation into any of Eqs. (86) or (89), we get

$$
\Phi\left(a_{0}\right) \equiv \frac{C a_{0}^{2}}{\exp \left(-\frac{\varkappa a_{0}^{2}}{2}\right)-1}+\frac{2 C}{\varkappa}+C \frac{a_{0}^{2}}{2}+\frac{2 \varkappa}{m} \frac{F_{0}}{\pi a_{0}^{2}}=0 .
$$

We have obtained here the transcendental equation to determine unknown value $a_{0}=a(0)$. One can directly show that $\Phi(\xi) \rightarrow+\infty$ as $\xi \rightarrow+0, \Phi(\xi) \rightarrow-\infty$ as $\xi \rightarrow+\infty$ and $\Phi^{\prime}(\xi)<0$ for any $\xi>0$. This means that there exists the unique solution $a_{0}>0$ to equation (91).

On the other hand, one can determine the value of $a_{0}$ using a formally different routine. Namely, from Eq. (25) at $t=0$ it follows that

$$
\frac{\pi m}{48} C a_{0}^{6}=F_{0}+\varkappa\left(\frac{a_{0}^{2}}{4} F_{0}-2 \pi \int_{0}^{a_{0}} p(\rho, 0) \rho^{3} d \rho\right),
$$

In view of (87) after computation of the integrals, we get

$$
\frac{\varkappa}{m} \int_{0}^{a_{0}} p^{-}(\rho, 0) \rho^{3} d \rho=-C \frac{a_{0}^{6}}{24}+\left(\frac{2 C}{\varkappa}+\delta_{0}\left(0^{+}\right)\right)\left[\frac{a_{0}^{4}}{16}+\frac{a_{0}^{2}}{4 \varkappa} e^{-\varkappa \frac{a_{0}^{2}}{2}}-\frac{1}{2 \varkappa^{2}}\left(1-e^{-\varkappa \frac{a_{0}^{2}}{2}}\right)\right] .
$$

Finally, after some algebra, with taking into account Eqs. (86) and (89), one can check that (92) and (93) lead to Eq. (91).

If we consider the limit $\varkappa \rightarrow 0$, all three conditions will degenerate to the only one

$$
\delta_{0}(0)=\frac{C}{2} a_{0}^{2}
$$

coinciding with that obtained in Ateshian et al. (1994); Wu et al. (1997a). Passing to the same limit as $\varkappa \rightarrow 0$ in formula (85), we obtain

$$
p^{-}(r, 0)=m\left\{\delta_{0}(0) \frac{a_{0}^{2}-r^{2}}{4}-C \frac{a_{0}^{4}-r^{4}}{16}\right\} .
$$

Now, taking into account (94), we rewrite the latter equation in the form

$$
p^{-}(r, 0)=\frac{m \delta_{0}(0)}{8 a_{0}^{2}}\left(a_{0}^{2}-r^{2}\right)^{2} \equiv \frac{m}{16} C\left(a_{0}^{2}-r^{2}\right)^{2}
$$

that again corresponds to the result obtained previously by Ateshian et al. (1994). 


\section{A5. Long-time asymptotics}

Finally, in order to prove that we have constructed the uniformly valid asymptotic solution for the contact radius, let us verify that the solution of the approximate equation (16) satisfies the asymptotic condition (53). In order to simplify the considerations, we assume that $F(t)=F_{0}$, where $F_{0}=$ const. Then, formula (53) takes the form

$$
a(t) \sim c t^{1 / 6}, \quad t \rightarrow \infty
$$

Here and afterwards, $c$ denotes the constant which does not depend on $t$.

Consequently, the following asymptotic conditions must hold

$$
\mathcal{K} F(t) \sim c t, \quad a^{2}(t) F(t) \sim c t^{1 / 3}, \quad t \rightarrow \infty .
$$

and one can immediately conclude that the first term in the right-hand side of Eq. (16) is the dominant one as $t \rightarrow \infty$ under the asymptotic condition (96).

Further, integrating by parts, we obtain

$$
\mathcal{K}^{-1} g(t) \sim \int_{0}^{t} g^{\prime}(\tau) e^{-\chi(t-\tau)} d \tau, \quad t \rightarrow \infty .
$$

Hence, taking account of the asymptotic condition (96), we find

$$
\mathcal{K}^{-1} a^{8}(t) \sim c \int_{0}^{t} \tau^{1 / 3} e^{-\chi(t-\tau)} d \tau, \quad t \rightarrow \infty
$$

Integrating by parts in the integral in (99), we get

$$
\int_{0}^{t} \tau^{1 / 3} e^{-\chi(t-\tau)} d \tau=\frac{t^{1 / 3}}{\chi}-\frac{e^{-\chi t}}{3 \chi} \int_{0}^{t} \frac{e^{\chi \tau}}{\tau^{2 / 3}} d \tau
$$

The last integral can be estimated as follows:

$$
\begin{aligned}
& \chi^{1 / 3} \int_{0}^{t} \frac{e^{\chi \tau}}{\tau^{2 / 3}} d \tau=\int_{0}^{\chi t} \frac{e^{x}}{x^{2 / 3}} d x=\int_{0}^{1} \frac{e^{x}}{x^{2 / 3}} d x \\
& +\int_{1}^{\chi t} \frac{e^{x}}{x^{2 / 3}} d x \leq e \int_{0}^{1} \frac{1}{x^{2 / 3}} d x+\int_{1}^{\chi t} e^{x} d x=2 e+e^{\chi t} .
\end{aligned}
$$

Finally, taking into account both relations, we obtain

$$
\mathcal{K}^{-1} a^{8}(t) \sim c t^{1 / 3}, \quad t \rightarrow \infty
$$


The asymptotic formula (100) implies that the first term in the round brackets (14) is a dominant one as $t \rightarrow \infty$ under the asymptotic condition (96). This proves that the approximate equation (16) presents the uniformly valid asymptotic approximation to the equation for the radius of the contact area $(25)$ in the whole time range interval $t \in[0,+\infty)$. By an argument similar to the one above we can consider the general case of monotonic loading when the function $F(t)$ increases with time.

The same line of the reasoning can be provided for the equation (58). The behavior at infinity gives the same agreement whereas condition at the initial point, $t=0$, reads now

$$
\frac{\pi m C}{48 F(0)} a_{0}^{6}=\frac{240+60 \varkappa a_{0}^{2}+10 \varkappa^{2} a_{0}^{4}}{240+60 \varkappa a_{0}^{2}+9 \varkappa^{2} a_{0}^{4}} .
$$

Together with the conclusions from Section 4.3, this an additional explanation why the first order approximation (59) has not contained a term of the order $O(\varkappa)$. Notice that the rational approximation (101) coincides with that reported in (52) with accuracy to $O\left(\varkappa^{3}\right)$. 
Complete set of figures $(1-5)$

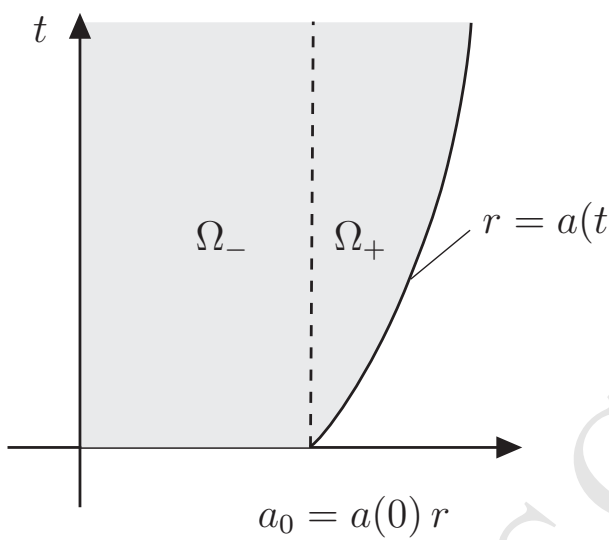

Figure 1. Support of the solution of Eq. (27). 

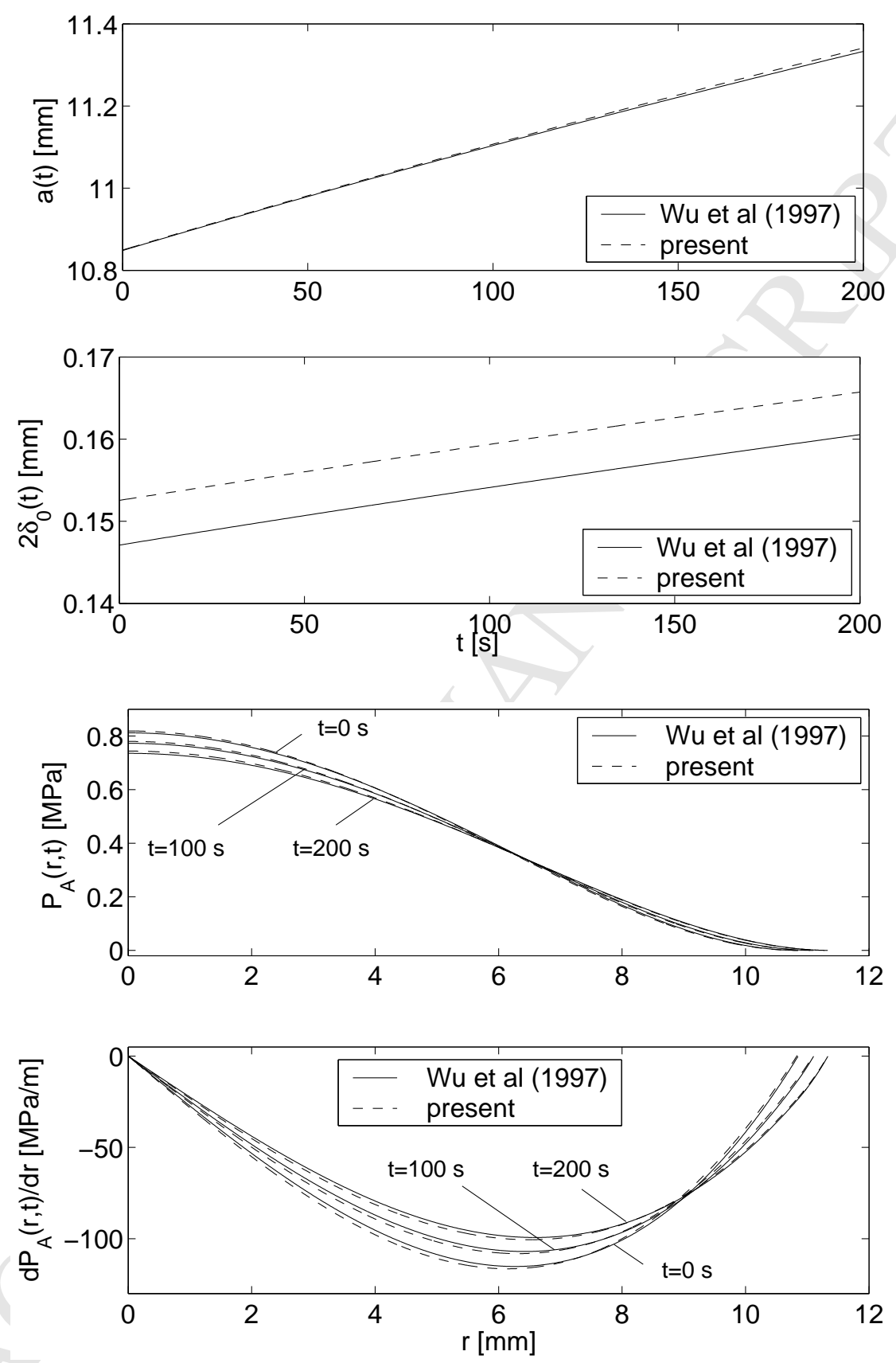

Figure 2. Prediction of the radius of the contact zone, $a(t)$, indentation $2 \delta_{0}(t)$ as functions of time and the distribution of the contact pressure $p(r, t)$ and its derivative with respect to the radial coordinate $r$ for three different moments of time $t=0,100,200[\mathrm{~s}]$ according to the model reported by Wu et al. (1997a) and the present one for the set of parameters according to (Ateshian et al., 1994). (See the first set of parameters in Table 1). 

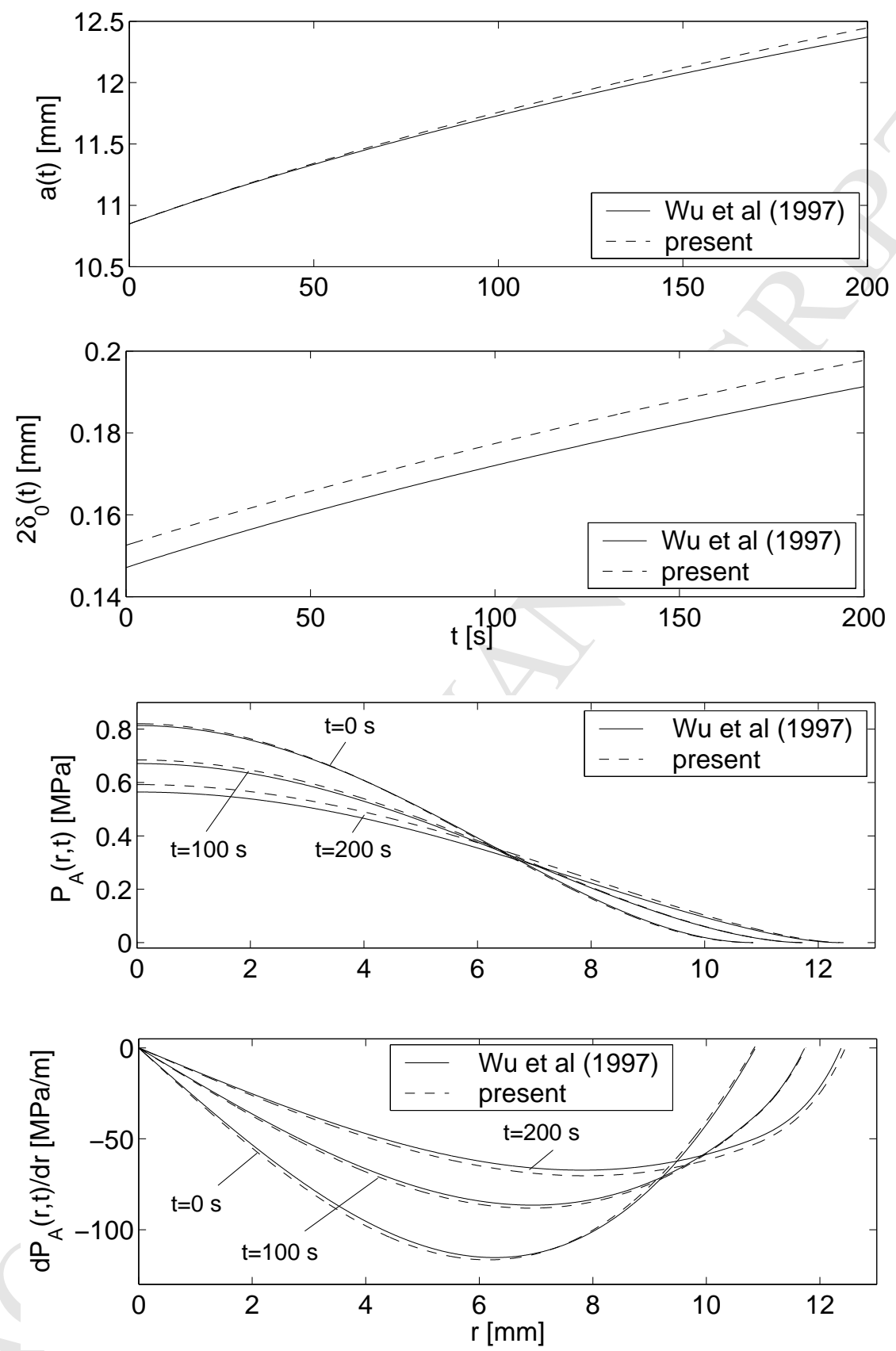

Figure 3. Prediction of the radius of the contact zone, $a(t)$, indentation $2 \delta_{0}(t)$ as functions of time and the distribution of the contact pressure $p(r, t)$ and its derivative with respect to the radial coordinate $r$ for three different moments of time $t=0,100,200[\mathrm{~s}]$ according to the model reported by Wu et al. (1997a) and the present one for the set of parameters: $k^{\prime}=4 k$ and others according to (Ateshian et al., 1994). (See the second set of parameters in Table 1). 

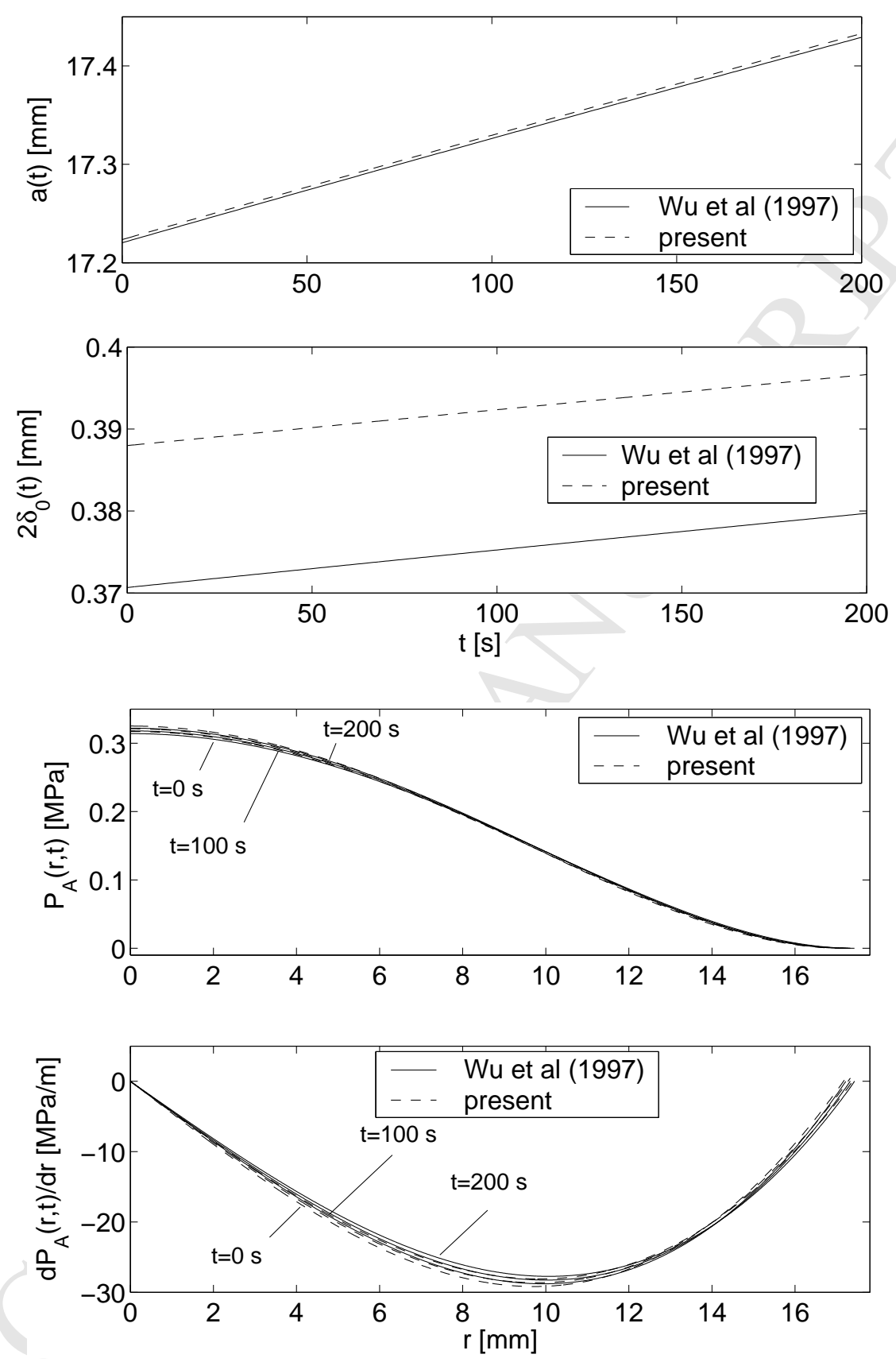

Figure 4. Prediction of the radius of the contact zone, $a(t)$, indentation $2 \delta_{0}(t)$ as functions of time and the distribution of the contact pressure $p(r, t)$ and its derivative with respect to the radial coordinate $r$ for three different moments of time $t=0,100,200[\mathrm{~s}]$ according to the model reported by Wu et al. (1997a) and and the present one for the set of parameters: $h^{\prime}=2 h$, $k^{\prime}=2 k, \mu_{s}^{\prime}=0.5 \mu_{s}$, while others according to (Ateshian et al., 1994). (See the third set of parameters in Table 1 ). 

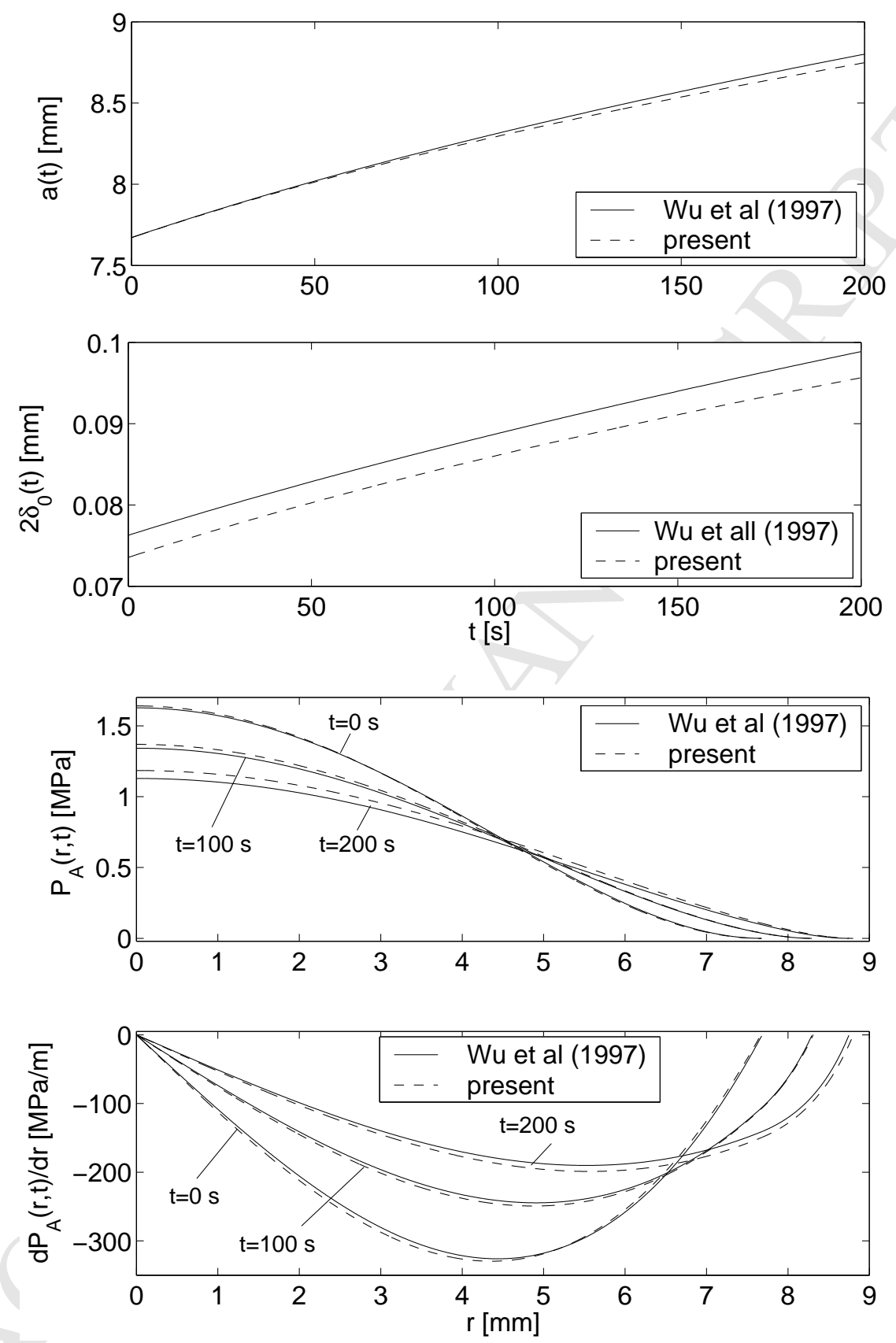

Figure 5. Prediction of the radius of the contact zone, $a(t)$, indentation $2 \delta_{0}(t)$ as functions of time and the distribution of the contact pressure $p(r, t)$ and its derivative with respect to the radial coordinate $r$ for three different moments of time $t=0,100,200[\mathrm{~s}]$ according to the model reported by Wu et al. (1997a) and and the present one for the set of parameters: $h^{\prime}=h / 2$ and others according to (Ateshian et al., 1994). (See the fourth set of parameters in Table 1). 